\title{
Welfare-Maximizing Assignment of Agents to Hierarchical Positions
}

\author{
Isa Hafalir and Antonio Miralles*
}

September 8, 2015

\begin{abstract}
We allocate agents to three kinds of hierarchical positions: top, medium, and low. No monetary transfers are allowed. We solve for the incentive-compatible (IC) mechanisms that maximize a family of weighted social welfares that includes utilitarian and Rawlsian welfares. When the market is tough (all agents bear positive risk of obtaining a low position in any IC and feasible mechanism), then the pseudomarket mechanism with equal budgets (PM) and the Boston mechanism without priorities (BM) yield identical assignments which are always optimal. Otherwise, when the market is mild, PM and $\mathrm{BM}$ differ and each one implements the optimal rule under different assumptions on the curvature of virtual valuations. We also establish that both BM and PM mechanisms guarantee IC Pareto-optimal assignments for a domain of preference distributions satisfying weak assumptions.

Keywords: Mechanism design, Welfare maximization, Incentive-compatible Pareto frontier, No transfers, Assignment problems, Pseudomarket, Boston mechanism.

JEL Codes: D82, C72, C78.

*Affiliations: Carnegie Mellon University (Hafalir and Miralles) and Universitat Autonoma de Barcelona - BGSE (Miralles). Emails: isaemin@cmu.edu (Hafalir) and antonio.miralles@uab.cat (Miralles). Hafalir thanks Universitat Autonoma de Barcelona for hosting him during the early stages of the project, and acknowledges financial support from National Science Foundation grant SES-1326584. Antonio Miralles acknowledges financial support, from the Ramón y Cajal contract of the Spanish Ministerio de Ciencia y Tecnologia, from the Spanish Plan Nacional I+D+I (ECO2014-53051-P), the Generalitat de Catalunya (SGR2014505) and the Severo Ochoa program (SEV2011-0075). We would like to thank Andreas Blum, Johannes Gierlinger, Johannes Horner, Ali Hortacsu, Marek Pycia, Tomás Rodríguez-Barraquer, Tayfun Sönmez, Utku Ünver, and seminar participants in University of Arizona, Sabanci University, UC Berkeley, and Caltech for their useful comments and suggestions.
\end{abstract}




\section{Introduction}

In a seminal paper, Hylland and Zeckhauser (1979) take a market design perspective on the problem of assigning positions to agents, where monetary transfers are not allowed and each agent is eventually assigned to exactly one position. Their model is motivated by important real-world applications such as assignment of legislators to committees, faculty members to offices, students to public schools, and workers to positions, departments, or tasks in various organizations and firms. These environments can be modeled as "assignment games" in which (i) agents and positions are indivisible (yet probabilistic assignment to positions are allowed); (ii) preferences of agents over positions may be their private information, and hence agents can respond to mechanisms strategically rather than truthfully, and; (iii) a medium of exchange such as money, for various reasons, is not an acceptable instrument. Hylland and Zeckhauser (1979) introduce the "Pseudomarket mechanism," in which the individuals use "fake money" to buy assignment probabilities for different kinds of positions. The Pseudomarket has good efficiency properties. More specifically, it is "ex-ante" efficient in the sense that there cannot be any other probabilistic assignment that would make all the agents better off. ${ }^{1}$

In this paper, we take a mechanism design approach to the same problem, i.e., we solve a cardinal symmetric mechanism design ${ }^{2}$ problem in order to maximize important social welfare criteria when transfers are not available. The basic idea is that, although there are no monetary transfers, allocation probabilities of different objects can still be used for representing "allocation and transfer rules" in the standard mechanism design problem. Then we can utilize standard techniques (such as envelope results) in mechanism design, except with one important complication: in our model, since the transfer rule is a probability, it has to be between 0 and 1; whereas in the standard problem transfers can take any values. We use novel methods in order to cope with this complication.

We study a continuum economy ${ }^{3}$ (with unit mass of players and positions) with three kinds of positions which agents rank equally: top, medium and low. In the discussion section we also consider a case where agents' ordinal preferences may be different from each other. The main reason that we focus on three kinds of positions is to be able to work on "single-dimensional" private information. Multi-dimensional optimal mechanisms, even in the case with transfers, are well-known to pose a very difficult problem. In this context, Armstrong (2000) illustrates how cumbersome the problem becomes when we consider revenue-maximizing optimal mechanisms. ${ }^{4}$ More recently, a series of papers by Hart and coauthors (Hart and Reny, 2010; Hart and Reny, 2012, Hart and Nisan, 2012) argue that the general problem becomes practically unsolvable when there is more than one good for sale and analyze if simple mechanisms perform well in terms of revenue.

To motivate our model with three types of positions and a continuum of players, consider a society or organization that cannot provide the same social or hierarchical status to all of its members. Naturally, every member prefers belonging to the highest position and dislikes being relegated to the lowest ladder of the

\footnotetext{
${ }^{1}$ Moreover, it does not generate envy when all agents face the same budget limit. The converse is true in atomless economies with a continuum of agents, from Thomson and Zhou (1993): any efficient and envy-free random assignment can be obtained through a Pseudomarket equilibrium with equal budgets.

${ }^{2}$ Throughout the whole paper we restrict attention to symmetric mechanisms. Any two agents of the same type would obtain the same random assignment.

${ }^{3}$ The mechanisms we highlight here, above all the Pseudomarket mechanism, make special sense in big assignment economies, where a single agent may have a negligible impact on market conditions.

${ }^{4}$ Altough our criteria is "welfare maximization," and not "revenue maximization." In the environments without transfers, VCG type of mechanisms cannot be used, and by incorporating incentive-compatibility conditions on the objective function, one would need to solve a problem which resembles very much that of a revenue maximizing optimal mechanism.
} 
hierarchy. However, agents differ in several dimensions of personality and abilities to complete tasks attached to every position. This determines some differences in the intensity of their preferences. For instance, riskaverse or conforming agents might prefer taking to a medium position with certainty rather than facing a lottery over either being assigned to a high position or being assigned to a low position. The opposite would be true for less risk-averse or more ambitious agents. In general, same phenomena hold for the allocation of resources or tasks in various organizations. ${ }^{5}$ This postulates a mechanism design problem: how do we distribute agents to the available positions so that we optimize a given notion of social welfare?

We focus on a family of social welfare functions that include utilitarian welfare (maximizing the unweighted average of all agents' expected payoffs) and Rawlsian welfare (maximizing the minimum payoff). In a nontransferable utility environment, we partially characterize the incentive-compatible (IC) Pareto efficiency frontier ${ }^{6}$ by considering various weighted social welfare functions. We pay additional attention on stronger efficiency notions (utilitarian and Rawlsian) following Harsanyi (1955) and the "veil of ignorance" (no agent knows in advance her type and attaches the corresponding probabilities to each different type). If the agents are expected utility maximizers, utilitarian welfare (à la Harsanyi, 1955) is the best choice. If, on the other hand, the agents are extremely pessimistic, Rawlsian welfare (à la Rawls, 1972) is the best choice.

Interestingly, we find that the mechanisms implementing the optimal assignment rules are widely known in the literature on matching and assignment problems. Either the pseudomarket with equal budgets (PM) or the widely debated Boston mechanism with no preexisting priority rights $(\mathrm{BM})^{7}$ turns out to be optimal for a variety of cases, depending on the distribution of cardinal preferences.

To cope with interpersonal comparability, we adopt a double normalization such that being assigned to top positions gives unit payoff, while being assigned to low-class positions gives zero utility. Being assigned a medium-class position gives a utility $v \in[0,1]$, which is the private information of the agents. In other words, we measure the agent's degree of success, where getting the most-preferred position entails total success, getting a low-class position entails total failure, and obtaining a medium position implies a variable degree of success, depending on how the agent values it. In the extension subsection 4.1, we suppose that top position gives a certain payoff $a \in(0,1)$, and medium position values are still in $[0,1]$, therefore medium positions can be strictly preferred to top (or certain) position in this extension. We find that similar results hold for this case as well, and therefore demonstrate that our results are not an artifact of "same ordinal rankings" among the types of objects.

The distribution function of this parameter $v, F(\cdot)$, with positive, differentiable density $f(\cdot)$, characterizes the demand side of this economy. We make the auction theory assumptions that both the "seller"- and "buyer"-virtual valuations (respectively $H(\cdot)$ and $J(\cdot)$ ) are increasing functions (Assumption 1). The supply side is characterized by the limited capacities for high- and medium-class positions, respectively $\mu_{1}$ and $\mu_{2}$, where $\mu_{1}, \mu_{2}, \mu_{1}+\mu_{2} \in(0,1)$. For $p(v)$ denoting the probability that a $v$-type agent is assigned to top positions and $q(v)$ denoting her probability of being assigned to medium-class positions, incentive compatibility implies that $p(\cdot)+q(\cdot)$ is increasing and $p(\cdot)$ is decreasing. We show that a family of constraints

\footnotetext{
${ }^{5}$ For instance, in faculy-office assignments, it is reasonable to assume each faculty dislikes offices with no windows, likes bigger offices, but has a different intensity while comparing medium and big size offices.

${ }^{6}$ That is, the set of IC and feasible assignment rules that are not Pareto-dominated by any other IC and feasible assignment rule. In this paper, we restrict attention to symmetric mechanisms. Observe that IC and envy-freeness are equivalent in this model, hence every conclusion in the paper naturally extends to the envy-free Pareto frontier.

${ }^{7}$ See Abdulkadiroğlu and Sönmez (2003), Ergin and Sönmez (2006), and Pathak and Sönmez (2008), among others.
} 
-that $p(\cdot)$ and $q(\cdot)$ are proper probabilities- are reduced to two single constraints: the upper-bound (UB) constraint $p(1)+q(1) \leq 1$ and the lower-bound (LB) constraint $p(1) \geq 0$ (Proposition 2). These two additional constraints methodologically distinguish this problem from a standard mechanism design (e.g. auctions) problem.

Depending on the weight structure $\Omega$ we put on our social welfare function (where $\Omega(v)$ is the cumulative weight for agents with types weakly below $v$ ), and after considering incentive compatibility and feasibility constraints, social welfare becomes a linear functional of $K(\cdot)$, where $K(v)$ is the contribution that assigning a marginal unit of $q(v)$ makes to the objective function. The exact form for $K(v)$ depending on $F$ and $\Omega$ is found in the main model (Definition 5 ). We call it type $v$ 's $\Omega$-virtual valuation. $K(v)$ reduces to $v$ for the utilitarian problem, and to buyers' virtual valuation $J(v)$ for the Rawlsian problem. We study social welfare functions such that $K(v)$ is increasing (Assumption 2). This includes the utilitarian and, given Assumption 1, the Rawlsian welfare functions. Subsection 4.2 discusses on non-monotonicities of $K(\cdot)$.

We define a tough market as an assignment problem where all incentive compatibility and market-clearing assignment rules bear some risk for all agents of ending up in a low-class position: UB cannot bind. Whether the market is tough or not is independent from the objective function, that is, from $K(\cdot)$. In a tough market, it turns out that LB binds at the optimal solution. In Theorem 1, we establish a surprising result. When the market is tough, (i) the optimal rule for all increasing $K(\cdot)$ (which includes the cases of utilitarian and Rawlsian optimization), (ii) the PM competitive equilibrium allocation, and (iii) the BM Bayesian Nashequilibrium allocation are equivalent to each other. ${ }^{8}$ In all cases, there is a cutoff $d^{*}$ such that all mid positions are evenly split among types above the cutoff, and all top positions are evenly split among types below. Unassigned agents are then assigned to low positions.

On the other hand, in a mild market, high-v agents may obtain certain allocation to medium-class positions at worst. Moreover, in the optimal solution this is indeed the case: UB binds. It turns out that the nature of the optimal allocation rule depends on the curvature of the (seller's) virtual valuation with respect to the $\Omega$-virtual valuation. If $H^{\prime}(\cdot) / K^{\prime}(\cdot)$ is increasing (which is equivalent to $H$ being convex in the utilitarian problem), the PM allocation is uniquely optimal. On the other hand, if $H^{\prime}(\cdot) / K^{\prime}(\cdot)$ is decreasing (which is equivalent to $H$ being concave in the utilitarian problem), the BM allocation is uniquely optimal. The proof (in Appendix A) contains two parts: 1) showing that $\mathrm{PM}(\mathrm{BM})$ is optimal among two-step rules given the curvature conditions, and 2) showing that curvature conditions induce a second-order stochastic dominance argument that rule out any other assignment rule. This second part is necessary, since one cannot simply take for granted that the optimal rule is a two-step rule in mild markets. For a counterexample see Appendix D.

When we further assume that $f(v) v+F(v)$ is nondecreasing in $v$ (Assumption 3), we get more insights on the part of the IC Pareto frontier in mild markets: we show that every IC and feasible rule such that UB binds is IC Pareto-optimal (in the sense that there is a weight structure $\Omega$ that makes each such rule optimal). Since UB binds in mild markets for both BM and PM as well, both PM and BM mechanisms

\footnotetext{
${ }^{8}$ Miralles (2008) previously established that in the tough market case with only one kind of underdemanded position, BM and PM obtain the same allocation. What is new in our result is that this allocation is optimal for a family of welfare functions including utilitarian and Rawlsian goals. Miralles (2008)'s main finding is superiority of BM over DA and it does not solve for a optimal mechanism design problem.
} 
guarantee an IC Pareto-optimal assignment for every $F$ satisfying Assumptions 1 and 3, regardless whether the market is tough or mild. ${ }^{9}$

Both optimal mechanisms, PM and BM, provide market-like trade-offs. PM is a competitive market that is designed to close in one round, since agents are constrained to buy a proper probability distribution. At other extreme, BM can be understood as a multiround competitive market. In each round, agents who were unassigned at the end of the previous round obtain a new budget with which they buy a probability bundle that does not add up to more than 1 . In both cases, spending token money on one position can reduce the chances for other positions. This is in sharp contrast with Gale and Shapley's (1962) well-known deferred acceptance (DA) algorithm, where betting on the most-preferred option does not harm the agent's chances at other positions. In the context with perfectly aligned ordinal preferences, it is known that DA implements a Pareto-pessimal assignment rule (Miralles, 2008; Abdulkadiroğlu, Che and Yasuda, 2011). That is, every other IC assignment rule weakly Pareto-dominates that provided by the dominant strategy equilibrium of DA. ${ }^{10} \mathrm{PM}$ and $\mathrm{BM}$ has been well-studied and discussed in the earlier literature and some good properties have been established. For instance, ex-ante optimality of PM is established in Hylland and Zeckhauser (1979) and-with perfectly aligned ordinal preferences-superiority of BM over DA is established in Abdulkadiroğlu, Che and Yasuda, 2011. Yet, to the best of our knowledge, no prior paper has shown "social welfare optimality" of these mechanisms.

Assignment problems are difficult to treat from the point of view of optimal mechanism design when the objective function is welfare maximization. ${ }^{11}$ In this line, Budish (2012) argues that the search for good properties using matching theory tools should be preferred to the search for an optimal solution. Despite agreeing with this, it is worth to investigate what optimal mechanism design can tell us about "good" assignment problems. What we found is that the optimal solution regards well-known mechanisms in the matching literature under a wide range of parameters. Our findings enrich the debate, while we acknowledge the importance of other features: preexisting priorities, precedence, nonstrategic players, etc.

The rest of the paper is organized as follows. We end this introduction by discussing the small literature that considers mechanism design approaches on assignment problem models (where monetary transfers are not allowed.) Section 2 introduces the model and the problem, for which we provide a solution. Section 3 analyzes the implementation of the optimal assignment rules. Section 4 discusses other assumptions of agents' preferences, other objective functions, and the failure of PM and BM being optimal. Section 5 concludes. Proofs are relegated to the Appendix A. Appendix B contains the discussion related to the case of varying ordinal preferences. Appendix C elaborates on the intuition behind the role of concavity/convexity of virtual valuations with respect to $\Omega$-virtual valuations in mild markets. Appendix D shows that the optimal rule is not always a two-step rule. This latter appendix thus illustrates that characterizing the IC Pareto frontier is not a straightforward task.

\footnotetext{
${ }^{9}$ This is not surprising for PM since it is known that it guarantees Pareto-efficiency among all possible rules (not only IC ones). This, however, is interesting for the BM assignment, which is not unconstrained Pareto-optimal in mild markets yet it turns out to be IC Pareto-optimal.

${ }^{10}$ Since there are no preexisting priority rights, DA coincides in its allocation with random serial dictatorship, which is also equivalent to top-trading cycles from random allocation (Abdulkadiroğlu and Sönmez, 1998). Moreover, DA coincides with Bogolmonaia and Moulin's (2001) probabilistic serial mechanism when the market is large (Che and Kojima, 2010), as does any strategy-proof, symmetric, and ordinarily efficient mechanisms (Liu and Pycia, 2014). These mechanisms are dominated by either PM or BM, not only in the utilitarian sense, but also in the Rawlsian max-min sense. That is, PM and BM provide not only an efficient solution but also a fair solution to the problem we analyze.

${ }^{11}$ When the criteria are ex-post or ex-ante efficiency, ordinal rules such as serial dictatorship or probabilistic serial (Bogomolnaia and Moulin, 2001) perform well. Yet, social welfare maximization is not necessarily achieved by these mechanisms.
} 
Related Literature From a methodological point of view, our paper solves a more complex mathematical problem than a standard auction design problem, for at least two reasons. First, the "numeraire" good, in our case the probability of being assigned to top-class positions, is globally constrained, since the capacity for top positions is limited. Second, the probability of being assigned to top- and medium-class positions must satisfy proper probability constraints for each agent. Namely, no probability can be negative, and the sum of these two probabilities cannot exceed 1.

The most closely related paper on this methodological issue is Miralles (2012). The key feature that is different in the present paper is that every agent must end up in exactly one position, whereas Miralles (2012) left the number of finally obtained objects unconstrained. Miralles's optimal mechanism involved an auction of the commonly less preferred object using evenly split probabilities of the commonly preferred object as individual budgets. It could happen that the winner's bid lied below her budget. Hence with some probability she would finally obtain both objects. For this reason, Miralles's optimal mechanism could not simply be transferred to the problem at hand in this paper. The one position per agent constraint has a bite on the optimal mechanism.

Other than Miralles (2012), there are only a few papers that solve for Bayesian incentive-compatible optimal mechanisms when transfers are not available. ${ }^{12}$ Pycia (2014) solves for the utilitarian maximal mechanism in an example where the best cardinal mechanism is unboundedly better than the best ordinal mechanism. Hashimoto (2014) describes a general class of mechanisms that are incentive compatible and that can approximate any efficient mechanism in a large market. ${ }^{13}$ Ashlagi and Shi (2013) is motivated by the 2012-2013 Boston school choice reform and studies a social planner's problem of optimally (in order to balance efficiency, equity and busing costs) allocating school seats without the ability to differentiate agents by charging prices or requiring costly effort. Ashlagi and Shi (2013)'s cardinal efficiency notion is "no ex-ante Pareto domination," which is different from our objective of social welfare maximization. BenPorath, Dekel, and Lipman (2012), as in our model, consider an assignment problem with no transfers where a principal needs to allocate an object to one of many agents. In their model the principal can check an agent's information at a cost. In this setup, optimal mechanisms are shown to be randomizations over "optimal favored-agent mechanisms." The main difference between our model and theirs is that we do not consider costly verification and we consider multiple objects rather than a single object. Also recently, dynamic mechanism design without money has been studied in Johnson (2014) and Guo and Hörner (2015), in which trade-offs occur between present and future payoffs. Pseudomarket mechanisms, where agents use fake budgets to bid against each other, might be efficient in this context as well. Gomes and Pavan (2015) study centralized many-to-many matching in markets where agents have private information about (vertical) characteristics that determine match values. In their model, the optimal matching rule takes the form of a threshold rule, with the threshold decreasing in type. The form of negative assortativeness they document is reminiscent in certain ways of the results in our paper. ${ }^{14}$

In a cheap talk model, Che, Dessein, and Kartik (2013) considers an environment where an agent advises a principal on selecting one of multiple projects or an outside option. The agent does not internalize the

\footnotetext{
${ }^{12}$ There is a vast literature that considers optimal mechanisms when transfers are available. In the context of position auctions (which are multi-item auctions with single dimensional private information), Edelman, Ostrovsky and Schwarz (2007) shows that "Generalized Second Price Auction" has an equilibrium that results in VCG allocation and payoff profile. More recently, Johnson (2013) uses a mechanism design framework to characterize how an intermediary can design profit-maximizing matching markets.

${ }^{13}$ We are grateful to an anoymous referee for pointing out these two references.

${ }^{14} \mathrm{We}$ are grateful to an associate editor for pointing this out.
} 
principal's value from the outside option. They show that for moderate outside option values, strategic communication is characterized by "pandering," i.e. biasing recommendation toward conditionally betterlooking project. In their main model they only optimize over "simple" communication mechanisms, yet, under some assumptions they also show that an optimal simple mechanism is optimal in the class of all mechanisms without transfers. Lastly, Börgers and Postl (2009) consider a model where two agents must select one of three alternatives. Their ordinal rankings are commonly known and diametrically opposed. The agents' utilities of the middle-ranked alternative are i.i.d., privately observed random variables. For this compromise problem with non-transferrable utility, they show the impossibility of implementing the firstbest. Although they are unable to solve for second-best analytically, they also determine some characteristics of second-best decision rules.

\section{Allocation to Ranked Positions}

There are three kinds of positions: top, medium, and low. Every agent's utility from obtaining a top position is 1 . Obtaining a low position gives zero utility. The medium position gives a utility of $v$, which is distributed over $[0,1]$ according to continuous distribution $F \in \mathcal{C}^{2}$ and strictly positive density $f$. The valuation for the medium position is agents' private information. There is a continuum of agents with a mass $1, \mu_{1}$ measure of top positions, $\mu_{2}$ measure of medium positions, and $1-\mu_{1}-\mu_{2}$ measure of low positions (of course, $\mu_{1}, \mu_{2}$, $\left.1-\mu_{1}-\mu_{2} \in(0,1)\right)$.

We consider cardinal mechanisms ${ }^{15}$ without transfers, and by the revelation principle we focus on direct truthful mechanisms.

Definition 1 An assignment rule is an integrable function $(p, q):[0,1] \rightarrow \Delta$ where the range is the tridimensional simplex. In this notation, $p(v)$ and $q(v)$ denote the probability of being assigned to high and medium positions, respectively (since everybody will be allocated a position, the probability of being assigned to a low position is $1-p(v)-q(v))$.

We have the following market-clearing conditions:

$$
\begin{aligned}
& \int_{0}^{1} p(v) f(v) d v=\mu_{1}, \\
& \int_{0}^{1} q(v) f(v) d v=\mu_{2},
\end{aligned}
$$

which need to be satisfied by any feasible rule. ${ }^{16}$ We say that a direct rule $(p(\cdot), q(\cdot))$ is feasible if it satisfies the above two market-clearing conditions, and $p(v), q(v), 1-p(v)-q(v) \in[0,1]$ for all $v \in[0,1]$.

\section{Virtual valuations}

\footnotetext{
${ }^{15}$ We often use the terms "assignment rule" or "rule" instead of mechanism. We use these terms interchangeably.

${ }^{16}$ Note that the third market-clearing condition $\int_{0}^{1}(1-p(v)-q(v)) f(v) d v=1-\mu_{1}-\mu_{2}$ is automatically satisfied.
} 
Definition 2 Denote buyers' virtual valuation by

$$
J(v)=v-\frac{1-F(v)}{f(v)}
$$

and sellers' virtual valuation by

$$
H(v)=v+\frac{F(v)}{f(v)}
$$

Assumption 1 We assume that $J$ and $H$ are increasing functions.

Assumption 1 is a mild assumption. $J$ being increasing is a standard assumption in mechanism design literature and satisfied by many widely used distribution functions. $H$ being increasing is a rather weak assumption; if $F$ is logconcave, ${ }^{17}$ that is, if $\frac{F}{f}$ is nondecreasing, then $H$ is obviously increasing. Many distributions widely used in the literature are logconcave: all power, normal, log-normal, Pareto, Weibull, Gamma, exponential, logistic, extreme value, Laplace, Chi distributions have logconcave CDF's, and thus also result in an increasing $H$.

\section{Incentive compatibility}

A rule is truthful (or incentive compatible, IC) if no agent can be better off by announcing any other type. That is, for all $v, v^{\prime} \in[0,1]$, the following condition needs to hold:

$$
p(v)+q(v) v \geq p\left(v^{\prime}\right)+q\left(v^{\prime}\right) v
$$

Define $U(v)=p(v)+q(v) v$. By the envelope theorem method, we first observe the following payoff equivalence result, whose proof is standard and skipped (for instance, see Milgrom and Segal, 2002).

Lemma 1 Incentive compatibility is equivalent to

$$
U(v)=p(0)+\int_{0}^{v} q(t) d t
$$

and $q$ increasing, $p$ decreasing, $q+p$ increasing (together called monotonicity conditions).

We omit the standard proof. Note that since $p$ and $q$ are probabilities and given monotonicity conditions, any feasible rule has to satisfy the following conditions: $q(0) \geq 0, q(1) \leq 1, p(1) \geq 0, q(1)+p(1) \leq 1$ (together called boundary conditions). The last two conditions are respectively called lower-bound constraint (LB) and upper-bound constraint (UB). From previous Lemma and the market clearing condition for top positions one can substitute out the $p$ function with

$$
p(v)=\mu_{1}+\int_{0}^{1} J(t) q(t) f(t) d t+\int_{0}^{v} q(t) d t-q(v) v,
$$

\footnotetext{
${ }^{17}$ For an excellent discussion on log-concave probability and its economic applications, see Bergstrom and Bagnoli (2005).
} 
Once again, this is a standard procedure in mechanism design. Hence an assignment rule $(p, q)$ can be regarded from now on only in terms of $q$, as long as it satisfies the following conditions. The proof of the following lemma is relegated to the Appendix. In the proof, first by Lemma (1) and changing the order of integration, we write $p(\cdot)$ as a function of $q(\cdot)$ and $F(\cdot)$. Second we show that $q(0) \geq 0, p(1)+q(1) \leq 1$ and $p(1) \geq 0$ implies the rest of the boundary constraints and rewrite these constraints as a function of $q(\cdot)$ and $F(\cdot)$ only. Finally, we show that $q$ being increasing implies the other monotonicity constraints.

Lemma 2 An assignment rule is incentive compatible and feasible if and only if

$$
\begin{aligned}
\int_{0}^{1} q(t) f(t) d v & =\mu_{2} \quad \text { (Market Clearing for mid positions - MC) } \\
\int_{0}^{1} H(t) q(t) f(t) d t & \leq 1-\mu_{1}(\text { Upper Bound Constraint - } \boldsymbol{U B}) \\
\int_{0}^{1} H(t) q(t) f(t) d t & \left.\geq q(1)-\mu_{1} \text { (Lower Bound Constraint }-\boldsymbol{L B}\right) \\
q(0) & \geq 0 \\
q & \text { is weakly increasing }
\end{aligned}
$$

Remark 1 The UB and LB constraints distinguish our problem from a standard mechanism design problem (e.g. optimal auction problem).

\subsection{Weighted Social Welfare}

Let $\Omega(v) \geq 0$ denote the cumulative weight that all agents with type weakly below $v$ have in the social welfare function. $\Omega(\cdot)$ is non-decreasing, continuously differentiable and it meets $\Omega(1)=1$. Defining weights in this way allows us to put all the weight into the lowest type, as it happens in the Rawlsian problem. ${ }^{18}$

The weighted social welfare is given by

$$
\begin{aligned}
W S W & =\int_{0}^{1} U(v) d \Omega(v) \\
& =\int_{0}^{1}(p(v)+q(v) v) d \Omega(v)
\end{aligned}
$$

subject to MC, UB and LB, which are given in Proposition 2.

Since $p(v)=\mu_{1}+\int_{0}^{1} J(t) q(t) f(t) d t+\int_{0}^{v} q(t) d t-q(v) v$, we can write $W S W$ as

$$
W S W=\int_{0}^{1}\left(\mu_{1}+\int_{0}^{1} J(t) q(t) f(t) d t+\int_{0}^{v} q(t) d t\right) d \Omega(v)
$$

and since $\Omega(1)=1$, we have

$$
W S W=\mu_{1}+\int_{0}^{1} J(t) q(t) f(t) d t+\int_{0}^{1}\left(\int_{0}^{v} q(t) d t\right) d \Omega(v)
$$

\footnotetext{
18 Since we focus on symmetric mechanisms and in any IC rule the utility is increasing, Rawlsian social welfare is equal to the utility of 0 type, hence $\Omega(v)=1$ for all $v \in[0,1]$.
} 
Finally, by changing the order of integration in the third term, we can write

$$
W S W=\mu_{1}+\int_{0}^{1}\left(t+\frac{F(t)-\Omega(t)}{f(t)}\right) f(t) q(t) d t .
$$

Definition $3 K(t) \equiv t+\frac{F(t)-\Omega(t)}{f(t)}, t \in[0,1]$, is the $\Omega$-virtual valuation of type $t$ given a weight structure $\Omega$.

Assumption 2 We assume that $K$ is an increasing function.

For utilitarian and Rawlsian social welfare functions we have $\Omega(t)=F(t)$ and $\Omega(t)=1$, respectively. For general weighted social welfare maximization problems, (even under Assumption 1) $K$ may fail to be increasing as illustrated in Subsection 4.2. Assumption 2 is used in our main results: we solve for the weighted social welfare maximizing mechanisms for $F$ and $\Omega$ that makes $K$ an increasing function. In this regard, we give a partial characterization of Pareto efficiency frontier of incentive compatible mechanisms by considering weighted social welfare functions that are consistent with $K$ being increasing. Importantly, those results have implications regarding the Incentive-Compatible Pareto frontier, where no such assumption is needed.

Some useful definitions are in order.

Definition 4 The problem given $\Omega$ consists of choosing a non-decreasing positive function $q(\cdot)$ to maximize $W S W$ subject to $M C, U B$ and $L B$.

Definition 5 An assignment rule $q$ is Incentive-Compatible (or IC) Pareto-optimal if there is $\Omega$ such that $q$ solves the problem given $\Omega$.

Utilitarian social welfare: In this case we have $\Omega(v)=F(v)$, thus $K(v)=v$, obviously accomplishing with Assumption 2. Utilitarian social welfare is then

$$
U S W=\mu_{1}+\int_{0}^{1} q(v) v f(v) d v
$$

where the last line follows from the second of the market clearance conditions.

Rawlsian social welfare: This welfare is equal to the lowest utility of the society. Since in any IC rule the utility is increasing, Rawlsian social welfare is equal to the utility of 0 type, hence $\Omega(v)=1$ for all $v \in[0,1]{ }^{19}$ Therefore $K(v)=J(v)$, where Assumption 1 implies Assumption 2. Rawlsian welfare is then equal to

$$
R S W=p(0)=\int_{0}^{1} q(v) J(v) f(v) d v
$$

\footnotetext{
${ }^{19}$ Since lowest type only cares about high positions, the Rawlsian problem looks as if this type could sell medium positions, obtaining "revenues" in the form of chances for a high position. It is not therefore not a coincidence that buyers' virtual valuation appears in the objective function, just as it does in revenue-maximizing auctions.
} 


\subsection{Achieving Highest Social Welfare}

A first step involves the quick analysis of a simplified problem where constraints LB and UB are ignored.

\subsection{The Relaxed Problem}

Let us consider the problem in which we consider only the first constraint, $\mathrm{MC}\left(\int_{0}^{1} q(t) f(t) d t=\mu_{2}\right)$. It is easy to show the following under Assumption 2:

Lemma 3 In the relaxed problem, the solution is given by

$$
q^{R *}(t)=\left\{\begin{array}{cc}
1 & t \in\left[v^{*}, 1\right] \\
0 & t \in\left[0, v^{*}\right)
\end{array}\right.
$$

for

$$
v^{*}=F^{-1}\left(1-\mu_{2}\right)
$$

The proof is very straightforward and relegated to the Appendix.

In what follows, we simplify $\int_{0}^{1} H(t) q^{* R}(t) f(t) d t$ at this optimal solution as

$$
\begin{aligned}
\int_{v^{*}}^{1} H(t) f(t) d t & =\int_{v^{*}}^{1}(t f(t)+F(t)) d t \\
& =\left.F(t) t\right|_{t=v^{*}} ^{t=1} \\
& =1-v^{*} F\left(v^{*}\right) \\
& =1-\left(1-\mu_{2}\right) F^{-1}\left(1-\mu_{2}\right) .
\end{aligned}
$$

Recall that, UB requires $\int_{0}^{1} H(t) q(t) f(t) d t \leq 1-\mu_{1}$ and LB requires $\int_{0}^{1} H(t) q(t) f(t) d t \geq q(1)-\mu_{1}$, and $q^{R *}(1)=1$. Hence, when

$$
1-\left(1-\mu_{2}\right) F^{-1}\left(1-\mu_{2}\right)=1-\mu_{1}
$$

or

$$
\left(1-\mu_{2}\right) F^{-1}\left(1-\mu_{2}\right)=\mu_{1}
$$

$q^{R *}(1)$ satisfies both UB and LB and therefore solves all problems accomplishing with Assumption 2. Hence, when (4) holds, we have a simple optimal solution. For the rest of the paper, we consider the case in which $\left(1-\mu_{2}\right) F^{-1}\left(1-\mu_{2}\right) \neq \mu_{1}$.

Next, consider the problem of maximizing

$$
\int_{0}^{1} H(t) q(t) f(t) d t
$$

subject to

$$
\int_{0}^{1} q(t) f(t) d v=\mu_{2} .
$$


Similar to the proof of Lemma 3, the solution can be shown to be $q^{R *}(t)$. This means that for all incentive compatible mechanisms that satisfy the market clearing condition, if

$$
\int_{v^{*}}^{1} q^{* R}(t) f(t) d t=1-\left(1-\mu_{2}\right) F^{-1}\left(1-\mu_{2}\right)<1-\mu_{1}
$$

or

$$
\mu_{1}-\left(1-\mu_{2}\right) F^{-1}\left(1-\mu_{2}\right)<0,
$$

then UB never binds. That is, when (5) holds, in any incentive compatible and feasible mechanism, we have to have $p(1)+q(1)<1 .{ }^{20}$ Since $p(\cdot)+q(\cdot)$ is increasing, this means that under (5) all agents bear positive risk of being assigned to low positions. We call such an economy a tough economy.

On the other hand, if

$$
\mu_{1}-\left(1-\mu_{2}\right) F^{-1}\left(1-\mu_{2}\right)>0
$$

then there exist incentive compatible and feasible mechanisms that can guarantee some agents top or medium positions. We call such an economy a mild economy.

We summarize this important discussion below.

Definition 6 We call an economy in which all agents bear the risk of being assigned to low positions in any incentive compatible and feasible mechanism as a tough economy. Equivalently, a market is tough if $\mu_{1}-\left(1-\mu_{2}\right) F^{-1}\left(1-\mu_{2}\right)<0$. We call an economy in which some agents can guarantee to be assigned to top or medium positions in some incentive compatible and feasible mechanism a mild economy. Equivalently, a market is mild if $\mu_{1}-\left(1-\mu_{2}\right) F^{-1}\left(1-\mu_{2}\right)>0$.

Hence $\mu_{1}-\left(1-\mu_{2}\right) F^{-1}\left(1-\mu_{2}\right)=0$ sets a boundary for our toughness and mildness definitions for an economy. To elaborate on this, observe that its left-hand side (LHS) is increasing in both capacities, $\mu_{1}$ and $\mu_{2}$. It also decreases if the distribution of valuations for middle-class positions becomes stronger in the first-order stochastic sense. In sum, tougher competitive conditions (both on the demand and on the supply sides) reduce the value of this LHS. This sets this specific bound: in a tough market, the LHS is negative, whereas in a mild market, the LHS is positive. We treat each case differently.

In the next section, we show that under a tough economy, the optimal solution has to be a particular two-step function that satisfies $q(0)=0$ and LB has to hold. Then we show that under a mild economy, UB has to hold in the optimal rule.

\subsection{Tough Economy}

In a tough economy $\left(\mu_{1}-\left(1-\mu_{2}\right) F^{-1}\left(1-\mu_{2}\right)<0\right)$, the optimal solution is given by the following proposition. Given Assumptions 1 and 2, this solution is optimal for all weighted social welfare functions (including utilitarian and Rawlsian).

\footnotetext{
${ }^{20}$ Recall that UB was obtained by requiring $p(1)+q(1) \leq 1$.
} 
Proposition 1 In a tough economy, the optimal solution is

$$
q^{T *}(t)= \begin{cases}\frac{\mu_{1}}{F\left(v^{* *}\right) v^{* *}} & t \in\left[v^{* *}, 1\right] \\ 0 & t \in\left[0, v^{* *}\right)\end{cases}
$$

for $v^{* *}$ that uniquely solves

$$
\frac{F(v) v}{1-F(v)}=\frac{\mu_{1}}{\mu_{2}}
$$

The proof is relegated to the Appendix. The intuition for the optimality this two-step rule in tough markets is as follows. Every non-two-step rule can be feasibly "ironed-out" by creating a new discontinuity point $a$ with a jump in $q$ around it (decreasing $q$ below $a$ and increasing $q$ above,) improving welfare. While this increases the value of the UB constraint, it does not matter because we know this constraint will not bind. Among the two-step rules where UB cannot bind (i.e. where all types bear some risk of being assigned to low positions), it easy to see that the best to do is to split all mid positions among types above some cutoff, and all top positions among types below that cutoff.

\subsection{Mild Economy}

In a mild economy $\left(\mu_{1}-\left(1-\mu_{2}\right) F^{-1}\left(1-\mu_{2}\right)>0\right)$, the optimal solution depends on the monotonicity of $H^{\prime}(\cdot) / K^{\prime}(\cdot)$. Given Assumptions 1 and 2, the solutions below are optimal.

Proposition 2 In a mild economy, we have:

(1) if $H^{\prime}(\cdot) / K^{\prime}(\cdot)$ is constant, all IC feasible rules where UB binds are optimal (including those that are optimal under (2) and (3)).

(2) if $H^{\prime}(\cdot) / K^{\prime}(\cdot)$ is strictly increasing, the optimal rule is

$$
q^{M *}(t)= \begin{cases}\frac{\mu_{2}}{1-F\left(d^{*}\right)} & t \in\left[d^{*}, 1\right] \\ 0 & t \in\left[0, d^{*}\right)\end{cases}
$$

for $d^{*} \in(0,1)$ that uniquely solves

$$
\left(1-\mu_{1}-d^{*} \mu_{2}\right) F\left(d^{*}\right)=1-\mu_{1}-\mu_{2} .
$$

(3) if $H^{\prime}(\cdot) / K^{\prime}(\cdot)$ is strictly decreasing, the optimal rule is

$$
q^{M * *}(t)=\left\{\begin{array}{lr}
1 & t \in\left[d^{* *}, 1\right] \\
1-\frac{1-\mu_{2}}{F\left(d^{* *}\right)} & t \in\left[0, d^{* *}\right)
\end{array}\right.
$$

for

$$
d^{* *}=\frac{\mu_{1}}{1-\mu_{2}}
$$


Remark 2 Notice that for the utilitarian problem we have $H^{\prime}(\cdot) / K^{\prime}(\cdot)=H^{\prime}(\cdot)$, and for the Rawlsian problem we have $H^{\prime}(\cdot) / K^{\prime}(\cdot)=H^{\prime}(\cdot) / J^{\prime}(\cdot)$.

The proof is relegated to the Appendix. In the proof of this proposition, we first study two-step rules such that types below some cutoff $c$ receive a probability bundle while types above receive another bundle. Given the UB constraint and the feasibility constraint, a two-step mechanism is fully characterized by this cutoff $c$ with valid values along a closed interval. Whether the objective function is increasing or decreasing in $c$ in the utilitarian case depends on the monotonicity of $H^{\prime}(\cdot) / K^{\prime}(\cdot)$. We show that no other feasible IC allocation rule can welfare-dominate the best two-step rule when $H^{\prime}(\cdot) / K^{\prime}(\cdot)$ is monotonic.

Remark 3 Importantly, one cannot simply restrict attention to two-step allocation rules without loss of generality. See Appendix D for an example.

Since concavity/convexity of $H$ with respect to $K$ is crucial in Proposition 2, we briefly explain the intuition on the role that concavity/convexity plays in what follows (this is extensively explained in Appendix C.) For simplicity, let us focus on the utilitarian problem. Seller's virtual valuation $H(v)$ is regarded as an information cost, a price for the quantity of medium-class positions allocated to each type. Hence the maximization problem looks like a consumer's optimal choice problem with a budget constraint (UB) and an additional constraint: that quantities attached to each type must add up to some constant, which represents the capacity of medium positions. The slope of prices (here the virtual valuations) with respect to payoffs (here the types) is crucial in the decision: decreasing slopes -concavity- push towards priming extreme types (both the low and the high ones), while increasing slopes - convexity- bring the opposite implication. In the paper, we show that the PM allocation puts the lowest possible mid-position quantities on extreme types among all valid rules, thus it is utilitarian-efficient under convex virtual valuations. The BM allocation puts the highest possible mid-position quantities on extreme types among all valid rules, thus it is utilitarianefficient under concave virtual valuations. A similar logic can be used for the general problem, yet in this case payoffs are represented by $\Omega$-virtual valuations $K(v)$ instead of types.

Moreover, we would like to emphasize that in the proofs for Propositions 1 and 2, since the optimal rules turn out to be "cutoff rules" rather than increasing and continuous rules, we use direct methods rather than using "optimal control" tools such as Euler equation.

\subsubsection{IC Pareto Frontier}

The following assumption allows us to make general insights on the part of the IC Pareto frontier.

Assumption 3 We assume that $f(v) v+F(v)$ is nondecreasing in $v$.

An equivalent assumption is found in Mierendorff (2009). All power distribution functions satisfy this assumption, as well as those with nondecreasing density, and with exponential densities $k e^{g(v)}$ where $g^{\prime}(v) v \geq$ -2 (which is a condition met by the truncated normal $N\left(\mu, \sigma^{2}\right)$ if $\mu+2 \sigma^{2} \geq 1$ or by the truncated exponential with parameter $\lambda \leq 2$ ), among others.

As a corollary to Proposition 2, we establish the following. 
Corollary 1 In mild markets, all IC feasible rules where UB binds are IC Pareto-optimal. That is, for each $q$ where $U B$ binds, there is $\Omega$ such that $q$ solves the problem given $\Omega$.

As noted in the introduction, since UB binds in mild markets for both BM and PM as well, both PM and BM mechanisms guarantee an IC Pareto-optimal assignment for every $F$ satisfying Assumptions 1 and 3 .

\section{Implementation via Pseudomarket and Boston Mechanism}

In this section, we solve for equilibria of Hylland and Zeckhauser (1979)'s pseudomarket with equal budgets $(\mathrm{PM})$ and Boston mechanism without priorities (BM) in our setup. We establish that, in a tough market, they both result in the unique optimal allocation. We also show that in a mild market, the competitive equilibrium of PM implements $q^{M *}$, and the Bayesian Nash equilibrium of BM implements $q^{M * *}$.

We first formally describe and analyze the equilibria of the two mechanisms. We also show the uniqueness of the equilibria. We finally compare the equilibrium assignments to those arising from the optimal mechanisms.

\subsection{Pseudomarket with Equal Budgets}

In Hylland and Zeckhauser (1979)'s pseudomarket with equal budgets (PM), all agents are endowed with the same budget, which is normalized to 1 . This budget consists of "fake money," and thus does not generate utility per se. It is used to purchase probability units of the available social positions. We normalize the price for low-class positions to 0 , and we denote the price for upper-class positions as $\pi_{1}$ and the price for medium-class positions as $\pi_{2}$. Without loss of generality, we set $\pi_{1}>\pi_{2}>0$. Also, we can argue that $\pi_{1} \geq 1$. This is because any other type of price vector would entitle all agents to get the high position and therefore could not possibly clear the market. Let $p\left(v, \pi_{1}, \pi_{2}\right)$ be the probability that a $v$-type agent buys in order to be assigned the upper-class position. Also, let $q\left(v, \pi_{1}, \pi_{2}\right)$ denote her purchased probability of a medium-class position. A $v$-type agent solves the problem

$$
\max _{p, q} p+q v \quad \text { s.t. } \pi_{1} p+\pi_{2} q \leq 1
$$

where $p, q \geq 0, p+q \leq 1$. The solution to the problem is unique for almost all types $v \in[0,1],{ }^{21}$ and it is denoted as $p^{*}\left(v, \pi_{1}, \pi_{2}\right)$ and $q^{*}\left(v, \pi_{1}, \pi_{2}\right)$.

A competitive equilibrium $(C E)$ is a price vector $\left(\pi_{1}^{*}, \pi_{2}^{*}, 0\right)$ that satisfies (i) $\int_{0}^{1} p^{*}\left(v, \pi_{1}^{*}, \pi_{2}^{*}\right) f(v) d v=\mu_{1}$ and (ii) $\int_{0}^{1} q^{*}\left(v, \pi_{1}^{*}, \pi_{2}^{*}\right) f(v) d v=\mu_{2}$. An associated CE assignment is composed of the functions $p^{*}\left(\cdot, \pi_{1}^{*}, \pi_{2}^{*}\right)$ and $q^{*}\left(\cdot, \pi_{1}^{*}, \pi_{2}^{*}\right)$.

Existence of CE is guaranteed from Hylland and Zeckhauser (1979). The following proposition solves for the $\mathrm{CE}$ of the PM, and it also establishes uniqueness of CE. In the proof, we analyze two cases: the

\footnotetext{
${ }^{21}$ For all the types except for $v=\frac{\pi_{1}}{\pi_{2}}$.
} 
first is when medium positions are expensive $\left(\pi_{2}^{*} \geq 1\right)$, and the second is when medium positions are cheap $\left(\pi_{2}^{*}<1\right)$. It turns out that the former case corresponds to a tough market, and the latter one corresponds to a mild market.

Proposition 3 In a tough market, the unique CE prices are

$$
\begin{aligned}
& \pi_{1}^{*}=\frac{F\left(v^{*}\right)}{\mu_{1}} \\
& \pi_{2}^{*}=\frac{1-F\left(v^{*}\right)}{\mu_{2}}
\end{aligned}
$$

where $v^{*}$ solves

$$
\frac{F\left(v^{*}\right) v^{*}}{1-F\left(v^{*}\right)}=\frac{\mu_{1}}{\mu_{2}} .
$$

In this CE, types $v<v^{*}$ obtain the probability bundle $(p, q, 1-p-1)=$

$$
\left(\frac{\mu_{1}}{F\left(v^{*}\right)}, 0,1-\frac{\mu_{1}}{F\left(v^{*}\right)}\right)
$$

and types $v>v^{*}$ get $(p, q, 1-p-1)=$

$$
\left(0, \frac{\mu_{2}}{1-F\left(v^{*}\right)}, 1-\frac{\mu_{2}}{1-F\left(v^{*}\right)}\right)
$$

(where the first, second, and third components represent probability shares of high, medium, and low positions, respectively).

On the other hand, in a mild market, the unique CE prices are

$$
\begin{aligned}
\pi_{1}^{*} & =\frac{F\left(v^{*}\right)}{F\left(v^{*}\right)-\left(1-\mu_{1}-\mu_{2}\right)} \\
\pi_{2}^{*} & =\frac{1}{\mu_{2}} \frac{\left(1-\mu_{1}\right) F\left(v^{*}\right)-\left(1-\mu_{1}-\mu_{2}\right)}{F\left(v^{*}\right)-\left(1-\mu_{1}-\mu_{2}\right)}
\end{aligned}
$$

where $v^{*}$ solves

$$
\left(1-\mu_{1}-v^{*} \mu_{2}\right) F\left(v^{*}\right)=1-\mu_{1}-\mu_{2}
$$

In this CE, the assignment of probabilities is as follows. Types $v<v^{*}$ obtain the probability bundle $(p, q, 1-p-1)=$

$$
\left(1-\frac{1-\mu_{1}-\mu_{2}}{F\left(v^{*}\right)}, 0, \frac{1-\mu_{1}-\mu_{2}}{F\left(v^{*}\right)}\right),
$$

and types $v>v^{*}$ get $(p, q, 1-p-1)=$

$$
\left(1-\frac{\mu_{2}}{1-F\left(v^{*}\right)}, \frac{\mu_{2}}{1-F\left(v^{*}\right)}, 0\right)
$$

\subsection{Boston Mechanism without Priorities}

In the Boston mechanism without priorities $(B M)$, agents simultaneously rank social classes. A round-byround algorithm serves to assign the available positions. In a first round, we consider each agent for the social class she ranked in the top position. When there is an excess of considered individuals with respect 
to available positions, a fair lottery defines who is accepted and who is rejected. If there is no such excess demand, every considered agent is accepted. An accepted agent obtains a position for which he was being considered. The rejected agents go to a second round in which they are considered for the social class they ranked second, and a similar acceptance/rejection procedure applies for the not-yet-assigned positions. After a finite number of rounds (three at most in this case), all agents are eventually accepted for some social class position. It is straightforward to see that no agent would rank the low-class position other than last. So the strategy space simplifies to two relevant strategies: either ranking the high class in first position (strategy 1 ) or ranking the medium class in first position (strategy 2). Let $m_{j}$ be the mass of agents using strategy $j \in\{1,2\}$.

In Proposition 4, we solve for the unique Bayesian Nash equilibrium of BM. It is straightforward to see that we have to have $m_{1}>\mu_{1}$. In the proof, as in the proof of Proposition 3, we analyze two cases: first when medium positions are overdemanded $\left(m_{2} \geq \mu_{2}\right)$ and second when medium positions are underdemanded $\left(m_{2}<\mu_{2}\right)$. It turns out that the former case corresponds to a tough market, and the latter corresponds to a mild market.

Proposition 4 In the unique Bayesian Nash equilibrium of BM, agents with values greater than $v^{*}$ use strategy 2, and agents with values smaller than $v^{*}$ use strategy 1. In a tough market, $v^{*}$ is the solution to

$$
\frac{F\left(v^{*}\right) v^{*}}{1-F\left(v^{*}\right)}=\frac{\mu_{1}}{\mu_{2}}
$$

whereas in a mild market,

$$
v^{*}=\frac{\mu_{1}}{1-\mu_{2}}
$$

In the Bayesian Nash equilibrium of the tough market, types $v<v^{*}$ obtain the probability bundle $(p, q, 1-p-1)=$

$$
\left(\frac{\mu_{1}}{F\left(v^{*}\right)}, 0,1-\frac{\mu_{1}}{F\left(v^{*}\right)}\right)
$$

whereas types $v>v^{*}$ get $(p, q, 1-p-1)=$

$$
\left(0, \frac{\mu_{2}}{1-F\left(v^{*}\right)}, 1-\frac{\mu_{2}}{1-F\left(v^{*}\right)}\right) .
$$

On the other hand, in the Bayesian Nash equilibrium of the mild market, types $v<v^{*}$ obtain the probability bundle $(p, q, 1-p-1)=$

$$
\left(\frac{\mu_{1}}{F\left(\frac{\mu_{1}}{1-\mu_{2}}\right)}, 1-\frac{1-\mu_{2}}{F\left(\frac{\mu_{1}}{1-\mu_{2}}\right)}, \frac{1-\mu_{1}-\mu_{2}}{F\left(\frac{\mu_{1}}{1-\mu_{2}}\right)}\right)
$$

whereas types $v>v^{*}$ get $(p, q, 1-p-1)=$

$$
(0,1,0)
$$

\subsection{Implementation of the Optimal Rules}

As a corollary to Propositions 1, 2, 3, and 4, we establish our main result, under Assumptions 1 and 2. The proof follows from noting that in a tough market both PM and BM result in allocation $q^{T *}$, and in a mild 
market PM results in allocation $q^{M *}$, whereas BM results in allocation $q^{M * *} .^{22,23}$ Point 3 ) in the Theorem below is a direct implication from the Corollary of Proposition 2.

Theorem 1 1) In a tough market, the optimal rule is implemented by both the competitive equilibrium of the pseudomarket with equal budgets and the Nash equilibrium in the Boston mechanism without priorities.

2) In a mild market, the optimal rule is implemented by competitive equilibrium of the pseudomarket if $H^{\prime}(\cdot) / K^{\prime}(\cdot)$ is increasing, and by the Boston mechanism without priorities if $H^{\prime}(\cdot) / K^{\prime}(\cdot)$ is decreasing.

3) Both BM and PM guarantee an IC Pareto-optimal assignment rule on the domain of preference distributions $F$ satisfying Assumptions 1 and 3.

\section{Discussion}

In the following subsections, we discuss several observations regarding the results of our model. First, we consider an extension of our model in which we allow agents to have varying ordinal preferences. Specifically, we suppose that the high position gives a constant utility $a \in(0,1]$ to every agent. In this extension, we show that PM and BM may still implement the optimal allocation if $a$ is not too low. Second, we illustrate that not all weighted utilitarian welfare functions have the same maximand in a tough market, if weights lead to non-monotonic $\Omega$-virtual valuations. Finally, we show that in a mild market neither the utilitariannor the Rawlsian-optimal assignments are always given by either PM or BM. With this we illustrate that the optimality of either PM or BM cannot be extended to every possible case. ${ }^{24}$

\subsection{Varying Ordinal Preferences}

A natural question is whether our result collapses if we relax our assumption that high-class positions are everyone's favorite. We work with the same model, but suppose that the high position gives a constant utility $a \in(0,1]$ to every agent. If $a$ is high enough (we do not require $a$ to be arbitrarily close to 1 ; we assume $\left.a \geq H\left(F^{-1}\left(1-\mu_{2}\right)\right)\right)^{25}$, that is, if high-class positions are still highly valued, we find the following facts:

1. Tough markets emerge "more easily" than when $a=1$, for fixed primitives $F, \mu_{1}$, and $\mu_{2}$. Intuitively, lower $a$ implies higher demand for mid-class positions; hence the likely impossibility of ensuring midclass positions to high types.

2. In tough markets, both $\mathrm{BM}$ and $\mathrm{PM}$ are still optimal, whenever $K(\cdot)$ is increasing.

\footnotetext{
${ }^{22}$ More formally, it is achieved by noting the equality between (i) $v^{* *}$ in (8) and $v^{*} \mathrm{~s}$ in (14) and (20), (ii) $q^{T *}$ in (7) and the second components of (15), (16), (22), and (23), (iii) $d^{*}$ in (10) and $v^{*}$ in (17), (iv) $d^{* *}$ in (12) and $v^{*}$ in $(21)$, (v) $q^{M *}$ in $(9)$ and the second components of (18), and (19), and finally (vi) $q^{M * *}$ in (11) and the second components of (24), and (25).

${ }^{23}$ Note that we only check for the probabilities of being allocated to the medium positions. This is sufficient because since the mechanisms are incentive compatible, payoff equivalence implies that the probabilities of being allocated to high or low positions are the same as well.

${ }^{24}$ Moreover, we can also show that in a mild market, the utilitarian- and the Rawlsian-optimal assignments may not coincide. For instance, it could be the case that BM provides the solution for utilitarian problem while PM provides the solution for Rawlsian problem. An example is available from the authors upon request.

${ }^{25}$ It is possible to achieve similar results for the case $a<H\left(F^{-1}\left(\mu_{2}\right)\right)$, yet the analyzed case is simpler to illustrate.
} 
3. In mild markets, a bias arises in favor of BM. Sufficient conditions for optimality of BM are still sufficient in this extended model. The intuition is similar to the one of the fact 1 above. Because medium positions are more appreciated overall, BM becomes more appealing than PM. The former guarantees certain assignment of medium positions to sufficiently high types, while the latter does not.

In Appendix B, we formalize these results.

Next, we demonstrate that in a tough market PM and BM may fail to maximize a weighted utilitarian social welfare-maximization problem.

\subsection{Nonmonotonic $\Omega$-virtual valuations}

In this subsection, we consider a weighted utilitarian objective where $\Omega$-virtual valuations $K(\cdot)$ are not always increasing. We demonstrate that even in a tough market, the optimal assignment may be different from PM and BM allocations.

Example 1 Consider $F(t)=t$ (so that $f(t)=1$ and $H(t)=2 t), \Omega(t)=t^{3}, \mu_{2}=0.2$, and $\mu_{1}=0.64$. For these specifications, $K(t)=2 t-t^{3}$.

Then, the relaxed problem in the weighted utilitarian case is to maximize

$$
\int_{0}^{1}\left(2 t-t^{3}\right) q(t) d t
$$

subject to

$$
\int_{0}^{1} q(t) d v=0.2 .
$$

The solution to this problem is given by the solution to the following problem

$$
\max _{c} \frac{0.1}{1-c} \int_{c}^{1}\left(2 t-t^{3}\right) d t .
$$

which has the solution of

$$
c=0.72076
$$

Hence, the optimal solution for the relaxed problem of weighted utilitarian case is

$$
q^{*}(t)= \begin{cases}0.71623 & t \geq 0.72076 \\ 0 & t<0.72076\end{cases}
$$

Moreover, since

$$
0.71623 \int_{0.72076}^{1} 2 t d t=0.34415,
$$

we can confirm that this solution also satisfies $U B$ and $L B(0.34415 \leq 1-0.64$ and $0.34415 \geq 0.71623-0.64)$. This is the solution to the original problem as well. 
Finally, for these specifications, we can quickly check that the optimal solution for the utilitarian and Rawlsian cases are different from the above solution. It is

$$
q^{*}(t)= \begin{cases}1 & t \geq 0.8 \\ 0 & t<0.2\end{cases}
$$

Next, we consider an explicit example that shows that in a mild market, the utilitarian and Rawlsian optimal assignments may differ from the allocations of PM and BM.

\subsection{Failure of PM and BM to Be Optimal in a Mild Market}

In Section 2.5, we gave sufficient conditions for PM or BM to be optimal. Now, we provide an explicit example in which neither PM nor BM is optimal in the utilitarian and Rawlsian maximization problems. To achieve this we consider an $F$ such that $H$ is neither convex nor concave. In the next example, the utilitarian and Rawlsian optimal mechanisms are different from both PM and BM allocations.

Example 2 Consider

$$
F(x)=\frac{x}{x^{3}-2 x^{2}+x+1} .
$$

Then can calculate that

$$
\begin{aligned}
H(x) & =\frac{x\left(-x^{3}+x+2\right)}{-2 x^{3}+2 x^{2}+1} \\
J(x) & =\frac{-x^{6}+4 x^{5}-7 x^{4}+2 x^{3}+4 x^{2}-1}{-2 x^{3}+2 x^{2}+1}
\end{aligned}
$$

and confirm that they are both increasing. Also, consider $\mu_{1}=0.3$, and $\mu_{2}=0.6$; then we have $d^{* *}=\frac{0.3}{0.4}=$ 0.75 , and $d^{*}$ solves $(0.7-0.6 x) \frac{x}{x^{3}-2 x^{2}+x+1}-0.1=0$, with numerical solution $d^{*}=0.19258$.

For this example, neither the PM allocation nor the BM allocation coincides with either the utilitarian optimal allocation or the Rawlsian optimal allocations. All are different from each other. Specifically, the PM allocation is a two-step function with cutoff approximately at $d^{*}=0.19258$, the BM allocation is a twostep function with cutoff at $d^{* *}=0.75$, the utilitarian optimal allocation can be calculated to be a two-step function with cutoff approximately at 0.523 , and the Rawlsian optimal allocation is can be calculated to be two-step function with cutoff at 0.5 .

\section{Conclusion}

Except for a few papers, examples of optimal mechanism design tools being used for the study of assignment problems are virtually nonexistent. In this paper, we consider an assignment problem with a continuum of agents and hierarchically ordered positions and we solve a family of weighted welfare maximization problems (which includes the utilitarian and Rawlsian welfare notions) subject to incentive constraints. Our results are surprising in that they provide arguments in favor of mechanisms that are well known and debated in 
the matching theory literature. For the first time that we are aware of, well-studied mechanisms such as the pseudomarket and Boston mechanisms are shown to be "second-best" welfare maximizers among all possible assignment rules (in the sense of being "first-best" among the incentive-compatible ones). It is also surprising that they are optimal not only in the utilitarian sense but also in the Rawlsian (max-min) sense. This is in sharp contrast to other mechanisms such as Deferred Acceptance, which works very poorly in this context.

We have also considered an extension of our model in which the ordinal rankings of the agents for positions may be different from each other. This extension highlights the fact that the results of the main model are not just an artifact of the same ordinal ranking assumption. ${ }^{26}$ In our model, we abstracted away from real-world features such as preexisting priorities and precedence. We plan to consider these issues further in future work. We hope that the results of the present paper will be a first step for more general and interesting results that make use of optimal mechanism design tools in economic environments without money.

\section{References}

1. Abdulkadiroğlu A. and Sönmez T. (1998) "Random Serial Dictatorship and the Core from Random Endowments in House Allocation Problems," Econometrica 66, 689-701.

2. Abdulkadiroğlu A. and Sönmez T. (2003) "School Choice, a Mechanism Design Approach," American Economic Review 93, 729-747.

3. Abdulkadiroğlu, Atila, Yeon-Koo Che, and Yosuke Yasuda. "Resolving Conflicting Preferences in School Choice: The Boston Mechanism Reconsidered." American Economic Review (2011): 399-410.

4. Armstrong M. (2000) "Optimal Multi-object Auctions," The Review of Economic Studies 67, 455-481.

5. Ashlagi, I. and Shi P. (2013) "Optimal Allocation of Public Services Without Monetary Transfers or Costly Signals," Working Paper, MIT.

6. Ben-Porath, E., E. Dekel, and B. Lipman "Optimal Allocation with Costly Verification." Working paper, Northwestern University, 2012.

7. Bergstrom, T, and Bagnoli, M. "Log-concave Probability and its Applications." Economic theory 26 (2005): 445-469.

8. Bogomolnaia A. and Moulin H. (2001) "A New Solution to the Random Assignment Problem," Journal of Economic Theory 100, 295-328.

9. Börgers, T. and Postl, P. (2009) "Efficient compromising." Journal of Economic Theory, 144(5), 20572076.

10. Budish E. (2012) "Matching 'versus' Mechanism Design," ACM SIGecom Exchanges 11, 4-15.

11. Che, Y., Dessein, W, and Kartik. N. "Pandering to persuade." The American Economic Review 103.1 (2013): 47-79.

\footnotetext{
${ }^{26}$ Note that in this extension, DA no longer implements a Pareto-pessimal assignment rule.
} 
12. Che Y. and Kojima F. (2010) "Asymptotic Equivalence of Probabilistic Serial and Random Priority Mechanisms," Econometrica 78, 1625-1672.

13. Edelman, B., Ostrovsky, M., Schwarz, M. (2007) "Internet advertising and the generalized second price auction: Selling billions of dollars worth of keywords." American Economic Review, 97 (1)

14. Ergin H. and Sönmez T. (2006) "Games of School Choice under the Boston Mechanism," Journal of Public Economics 90, 215-237.

15. Gale D. and Shapley L.S. (1962) "College Admissions and the Stability of Marriage," American Mathematical Monthly 69, 9-15.

16. Gomes, R. and Pavan A (2015) "Many-to-Many Matching and Price Discrimination." Working paper

17. Guo G. and Hörner J. (2015) "Dynamic Mechanisms without Money," manuscript.

18. Harsanyi J.C. (1955) "Cardinal welfare, individualistic ethics, and interpersonal comparisons of utility." The Journal of Political Economy, 63(4): 309-321.

19. Hart S., and Nisan N. (2012). "Approximate revenue maximization with multiple items." Working paper

20. Hart S., and Reny P. (2010) "Revenue maximization in two dimensions," Working paper

21. Hashimoto, T. (2013). "The generalized random priority mechanism with budgets." Working paper.

22. Hart S. and Reny P. (2012) "Maximal revenue with multiple goods: Nonmonotonicity and other observations." Working paper

23. Hylland A. and Zeckhauser R. (1979) "The Efficient Allocation of Individuals to Positions," Journal of Political Economy 87, 293-314.

24. Johnson T.R. (2013) "Matching Through Position Auctions", Journal of Economic Theory 148,17001713.

25. Johnson T.R. (2014) "Dynamic Mechanism Design Without Transfers: Promises and Confidentiality," Working paper

26. Krishna, V. (2002) "Auction Theory," Academic press.

27. Liu Q. and Pycia M. (2014) "Ordinal Efficiency, Fairness, and Incentives in Large Markets," Working paper

28. Mierendorff, K. (2009) "Optimal Dynamic Mechanism Design with Deadlines," Working paper

29. Milgrom, P., and Segal, I. (2002). "Envelope theorems for arbitrary choice sets." Econometrica, 70(2), 583-601.

30. Miralles A. (2008) "School Choice: The Case for the Boston Mechanism," Working paper

31. Miralles A. (2012) "Cardinal Bayesian Allocation Mechanisms without Transfers," Journal of Economic Theory 147, 179-206. 
32. Pathak P. and Sönmez T. (2008) "Leveling the Playing Field: Sincere and Sophisticated Players in the Boston Mechanism," American Economic Review 98, 1636-1652.

33. Pycia M. (2014) "The Cost of Ordinality," Working paper

34. Rawls, J. (1972) "A Theory of Justice." Oxford, Clarendon Press.

35. Thomson W. and Zhou L. (1993) "Consistent Allocation Rules in Atomless Economies," Econometrica $61,575-587$.

\section{Appendix A: Omitted Proofs}

Proof of Lemma 2. First of all, from Lemma (1), we know that incentive compatibility is equivalent to

$$
U(v)=p(0)+\int_{0}^{v} q(t) d t
$$

or

$$
q(v) v+p(v)=p(0)+\int_{0}^{v} q(t) d t
$$

Second, by the first market-clearing condition,

$$
\int_{0}^{1}\left(p(0)+\int_{0}^{v} q(t) d t-q(v) v\right) f(v) d v=\mu_{1}
$$

then, by changing the order of integration, we have

$$
p(0)=\mu_{1}+\int_{0}^{1} f(t) J(t) q(t) d t
$$

where

$$
J(t)=t-\frac{1-F(t)}{f(t)}
$$

is the virtual valuation.

Hence, function $p$ can be written solely as a function of $q$ :

$$
p(v)=\mu_{1}+\int_{0}^{1} f(t) J(t) q(t) d t+\int_{0}^{v} q(t) d t-q(v) v
$$

Note that we have incorporated the envelope condition and market-clearing condition for the top positions while writing this equality.

Therefore, a rule is incentive compatible and feasible if and only if it satisfies (i) equation (1), (ii) monotonicity conditions, (iii) boundary conditions, and (iv) the second market-clearing condition:

$$
\int_{0}^{1} q(t) f(t) d v=\mu_{2}
$$

Third, we argue that $p(1) \geq 0, p(1)+q(1) \leq 1$, and $q(0) \geq 0$ imply the rest of the boundary conditions: (i) $p(1)+q(1) \leq 1$ and $p(1) \geq 0$, then $q(1) \leq 1$; (ii) $p(1) \geq 0$ with monotonicity implies $p(0) \geq 0$; 
and $p(0) \geq 0$ and $q(0) \geq 0$ implies $q(0)+p(0) \geq 0$; and (iii) $p(1)+q(1) \leq 1$ and monotonicity implies $p(0)+q(0) \leq 1 ;$ and since $q(0) \geq 0$, this implies $p(0) \leq 1$.

We have

$$
\begin{aligned}
p(1) & =\mu_{1}+\int_{0}^{1} f(t) J(t) q(t) d t+\int_{0}^{1} q(t) d t-q(1) \\
& =\mu_{1}+\int_{0}^{1} f(t)\left(t-\frac{1-F(t)}{f(t)}\right) q(t) d t+\int_{0}^{1} q(t) d t-q(1) \\
& =\mu_{1}+\int_{0}^{1} f(t) t q(t) d t-\int_{0}^{1}(1-F(t)) q(t) d t+\int_{0}^{1} q(t) d t-q(1) \\
& =\mu_{1}+\int_{0}^{1} f(t) H(t) q(t) d t-q(1)
\end{aligned}
$$

where

$$
H(t)=t+\frac{F(t)}{f(t)}
$$

is the seller's virtual valuation or information rent.

Hence, $p(1) \geq 0$ is equivalent to

$$
\int_{0}^{1} H(t) q(t) f(t) d t \geq q(1)-\mu_{1}
$$

On the other hand, $p(1)+q(1) \leq 1$ is equivalent to

$$
\int_{0}^{1} H(t) q(t) f(t) d t \leq 1-\mu_{1}
$$

Finally, we argue that if $q$ is increasing, other monotonicity constraints ( $p$ is decreasing, $q+p$ is increasing) are automatically satisfied. Given $v>\tilde{v}$, incentive compatibility implies $p(\tilde{v})+q(\tilde{v}) \tilde{v} \geq p(v)+q(v) \tilde{v}$. Since $q(v) \geq q(\tilde{v})$ and $\tilde{v} \geq 0$, it must be the case that $p(\tilde{v}) \geq p(v)$. Also, incentive compatibility implies $p(\tilde{v})+q(\tilde{v}) v \leq p(v)+q(v) v$, or $(q(v)-q(\tilde{v})) v \geq p(\tilde{v})-p(v)$. Since $q$ is increasing, $p$ is decreasing, and $0 \leq v \leq 1$, we must have $q(v)-q(\tilde{v}) \geq p(\tilde{v})-p(v)$. This implies that $p+q$ is increasing.

To sum up, $p$ is uniquely determined given $q$, and the following conditions on $q$ are necessary and sufficient conditions for feasibility and incentive compatibility.

$$
\begin{gathered}
\int_{0}^{1} q(t) f(t) d v=\mu_{2} \\
\int_{0}^{1} H(t) q(t) f(t) d t \leq 1-\mu_{1} \\
\int_{0}^{1} H(t) q(t) f(t) d t \geq q(1)-\mu_{1}
\end{gathered}
$$

together with $q(0) \geq 0$ and $q$ is increasing.

Proof of Lemma 3. This is simply because marginal benefit of increasing $q(t)$ is $K(t) f(t)$. In both problems, the marginal cost is $f(t)$. The benefit-cost ratios are therefore given by $K(t)$. Since it is increasing in $t$, in the optimal solution, we should not allocate any positive $q$ 's to any $t$ 's unless all higher $\widehat{t}$ 's are already maxed out at $q(\widehat{t})=1$. Hence, the result obtains. 
Proof for Proposition 1. First of all, from the discussion before Definition 6, we can argue that UB never binds in a tough market. Hence, we can ignore UB.

Next, we establish the following lemma. In the proof, we first argue that the optimal solution has to be a "two-step" function by showing that an "ironed out" version of a non-two-step function gets a better welfare. Then we show that the lower step has to be 0 .

Lemma 4 The optimal solution for social welfare problems with $K(\cdot)$ increasing has to be of the form

$$
q(t)= \begin{cases}q(1) & t \in[d, 1] \\ 0 & t \in[0, d)\end{cases}
$$

Proof of Lemma 4 We first argue that optimal $q$ has to be a two-step function. Suppose not, that is, there exists an interval $[a, b]$ such that for all $t \in(a, b)$, we have $q(a)<q(t)<q(b)$. Then consider the "ironed out" version of $q$ in the interval $[a, b]$ :

$$
\widehat{q}(t)= \begin{cases}q(a) & \text { if } t \in[a, c] \\ q(b) & \text { if } t \in(c, b] \\ q(t) & \text { otherwise }\end{cases}
$$

for $c$ that satisfies

$$
q(a)(F(c)-F(a))+q(b)(F(b)-F(c))=\int_{a}^{b} q(t) f(t) d t .
$$

It is then immediate that $\widehat{q}$ satisfies MC. Also, one can check that $\int_{0}^{1} K(t) \widehat{q}(t) f(t) d t>\int_{0}^{1} K(t) q(t) f(t) d t$. Moreover, with the assumption that $H$ is an increasing function, we have $\int_{0}^{1} H(t) \widehat{q}(t) f(t) d t>$ $\int_{0}^{1} H(t) q(t) f(t) d t$. Therefore, $\widehat{q}$ also satisfies LB. Hence, we conclude that $q$ cannot be optimal. Then we argue that we only need to consider the following form of $q$ :

$$
\widetilde{q}(t)= \begin{cases}q(1) & t \in[d, 1] \\ 0 & t \in[0, d) .\end{cases}
$$

This is because if a there is a feasible two-step function of the form

$$
q(t)=\left\{\begin{array}{cc}
h & t \in[d, 1] \\
l & t \in[0, d)
\end{array}\right.
$$

for $h>l>0$ (note that $1>h$ since UB does not bind), we can find another two-step function

$$
q(t)= \begin{cases}h^{\prime} & t \in[d, 1] \\ l^{\prime} & t \in[0, d)\end{cases}
$$

with $1>h>h^{\prime}$, and $l>l^{\prime}$ that also satisfies the constraints and gives a higher objective function value for both problems. 
By Lemma 4, the maximization problem becomes

$$
\max _{d, q(1)} q(1) \int_{d}^{1} K(t) f(t) d t
$$

subject to the following two constraints.

$$
\begin{gathered}
q(1)(1-F(d))=\mu_{2} \\
q(1) \int_{d}^{1} H(t) f(t) d t \geq q(1)-\mu_{1} .
\end{gathered}
$$

Note that the second equality can be written as

$$
q(1)(1-F(d) d) \geq q(1)-\mu_{1}
$$

or

$$
\mu_{1} \geq q(1) F(d) d
$$

From the first constraint, we have

$$
q(1)=\frac{\mu_{2}}{(1-F(d))},
$$

which can be incorporated to the objective functions, making them

$$
\mu_{2} \mathbb{E}[K(V) \mid V \geq d]
$$

where $V$ represents the random variable $v$. They are both subject to

$$
\mu_{1} \geq \frac{\mu_{2}}{(1-F(d))} F(d) d
$$

Furthermore, note that both objective functions are increasing in $d$ and the right-hand side of the constraint is increasing in $d$. Therefore, optimal $v^{* *}$ uniquely solves

$$
\frac{F(d) d}{(1-F(d))}=\frac{\mu_{1}}{\mu_{2}}
$$

and optimal $q(1)^{*}$ satisfies

$$
q(1)^{*}=\frac{\mu_{2}}{\left(1-F\left(v^{* *}\right)\right)}=\frac{\mu_{1}}{F\left(v^{* *}\right) v^{* *}}
$$

Proof for Proposition 2. In the proof, we first show that UB has to bind at the optimal solutions, then solve for the maximizers among the two-step rules, then finally show that the two-step maximizer gives a higher social welfare than any $q$ for which UB binds.

We first establish the following lemma. In the proof, we first argue that if UB does not bind, then $q$ cannot be optimal unless it is a two-step function (again by considering an "ironed out" version of the function); then we argue that the maximizer among the two-step functions has to satisfy UB.

Lemma 5 UB has to bind at the optimal solution for all mild-market problems where $K(\cdot)$ is increasing. 
Proof of Lemma 5 First, we argue that if UB does not bind, then $q$ cannot be optimal unless it is a twostep function. Suppose $q$ is not a two-step function, that is, there exists an interval $[a, b]$ such that for all $t \in(a, b)$, we have $q(a)<q(t)<q(b)$. Then consider the "ironed out" version of $q$ in the interval $[a, b]:$

$$
\widehat{q}(t)= \begin{cases}q(a) & \text { if } t \in[a, c] \\ q(b) & \text { if } t \in[d, b] \\ q(t) & \text { otherwise }\end{cases}
$$

for $c$ and $d \in(a, b)$ that satisfies

$$
q(a)(F(c)-F(a))+q(b)(F(b)-F(d))=\int_{a}^{c} q(t) f(t) d t+\int_{d}^{b} q(t) f(t) d t .
$$

It is then immediate that $\widehat{q}$ satisfies MC. Also, one can check that $\int_{0}^{1} K(t) \widehat{q}(t) f(t) d t>\int_{0}^{1} K(t) q(t) f(t) d t$. Moreover, with the assumption that $H$ is an increasing function, we have $\int_{0}^{1} H(t) \widehat{q}(t) f(t) d t>$ $\int_{0}^{1} H(t) q(t) f(t) d t$. Since $\widehat{q}(1)=q(1)$, we can state that $\widehat{q}$ also satisfies LB. And for $c$ and $d$ close enough to $a$ and $d$, respectively, it continues to satisfy UB. Hence, we conclude that $q$ cannot be optimal.

Next, we argue that the maximizers among the two-step functions also should satisfy UB as equality. Consider a two-step function of the form

$$
q(t)=\left\{\begin{array}{cc}
k & t \in[c, 1] \\
l & t \in[0, c)
\end{array}\right.
$$

for $c \in[0,1], 0 \leq l \leq k \leq 1$.

Suppose UB does not bind; then $q(1)=k<1$. Consider $l=0$. Then the following assignment rule clearly increases the objective function's value

$$
q^{\prime}(t)= \begin{cases}k+\varepsilon & t \in\left[c+\varepsilon^{\prime}, 1\right] \\ l & t \in\left[0, c+\varepsilon^{\prime}\right)\end{cases}
$$

by choosing $\varepsilon, \varepsilon^{\prime}>0$ such that MC still holds, and $\varepsilon$, and $\varepsilon^{\prime}$ are small enough such that UB is still satisfied.

Moreover, we do not have to worry about LB in any two-step rule for a mild market when $l=0$. MC implies $k=\frac{\mu_{2}}{1-F(c)}$, and LB is written as $k(1-F(c) c) \geq k-\mu_{1}$. Joining both expressions we rewrite LB as $\frac{\mu_{2} F(c) c}{1-F(c)} \leq \mu_{1}$. Since $k \leq 1$ (or $c \leq F^{-1}\left(1-\mu_{2}\right)$ ) we have that the RHS of the previous inequality is not higher than $\left(1-\mu_{2}\right) F^{-1}\left(1-\mu_{2}\right)$, which is lower than $\mu_{1}$ in a mild market.

Now consider $0<l<k$. Then the following assignment rule also increases the objective function's value

$$
q^{\prime}(t)= \begin{cases}k & t \in\left[c-\varepsilon^{\prime}, 1\right] \\ l-\varepsilon & t \in\left[0, c-\varepsilon^{\prime}\right)\end{cases}
$$

by choosing $\varepsilon, \varepsilon^{\prime}>0$ such that MC still holds, and $\varepsilon$, and $\varepsilon^{\prime}$ are small enough such that UB is still satisfied. Since $q^{\prime}(1)=q(1)$ and $\int_{0}^{1} H(t) q^{\prime}(t) f(t) d t>\int_{0}^{1} H(t) q(t) f(t) d t, q^{\prime}$ also satisfies LB.

The final case involves $l=k=\mu_{2}$. It is easy to check that this uniform rule does not bind either UB 
or LB. The following rule

$$
q^{\prime}(t)= \begin{cases}\mu_{2}+\varepsilon & t \in\left[F^{-1}(1 / 2), 1\right] \\ \mu_{2}-\varepsilon & t \in\left[0, F^{-1}(1 / 2)\right)\end{cases}
$$

for small enough $\varepsilon>0$ meets MC, UB, and LB. $q^{\prime}$ clearly is an improvement over the uniform rule $q(t)=\mu_{2}$.

Hence, we have shown that no rule can be optimal if UB does not bind.

Hence, without loss of generality we consider assignment rules such that UB binds.

Let us solve for the maximizers among the two-step functions. That is, among the functions of the form

$$
q(t)=\left\{\begin{array}{cc}
h & t \in[c, 1] \\
l & t \in[0, c)
\end{array}\right.
$$

for $c \in[0,1], 0 \leq l<h \leq 1$.

Note that MC can be simplified as

$$
l F(c)+h(1-F(c))=\mu_{2}
$$

and that

$$
\int_{0}^{1} H(t) q(t) d t=l c F(c)+h(1-c F(c)) .
$$

Also, we have to have

$$
l c F(c)+h(1-c F(c))=1-\mu_{1}
$$

by Lemma 5 .

In a two-step rule with parameters $h, l, c$ where both MC and UB bind, we solve the equations (27) and (28) together for $h$ and $l$ and get

$$
\begin{aligned}
h(c) & =\frac{1-\mu_{1}-c \mu_{2}}{1-c} \\
l(c) & =\frac{1}{F(c)(1-c)}\left(F(c)+\mu_{2}+\mu_{1}-F(c) \mu_{1}-F(c) c \mu_{2}-1\right) \\
& =\frac{1-\mu_{1}-c \mu_{2}}{1-c}-\frac{1-\mu_{1}-\mu_{2}}{F(c)(1-c)} \\
& =h(c)-\frac{1-\mu_{1}-\mu_{2}}{F(c)(1-c)} \\
& \equiv h(c)-\frac{n(c)}{F(c)}
\end{aligned}
$$

$h(c) \leq 1$ implies

$$
1-c \geq 1-\mu_{1}-c \mu_{2}
$$

or

$$
c \leq \frac{\mu_{1}}{1-\mu_{2}}
$$


$l(c) \geq 0$ implies

$$
\left(1-\mu_{1}-c \mu_{2}\right) F(c) \geq 1-\mu_{1}-\mu_{2}
$$

Notice that $c$ is then acceptable if it lies on the interval $\left[d^{*}, \frac{\mu_{1}}{1-\mu_{2}}\right]$, where $d^{*}$ uniquely solves

$$
\left(1-\mu_{1}-d^{*} \mu_{2}\right) F\left(d^{*}\right)=1-\mu_{1}-\mu_{2} .
$$

Also notice that the lowest $c$ gives the rule $q^{M *}$ stated in the proposition, while the highest $c$ provides $q^{M * *}$.

Let us denote the objective function among the two-step functions with cutoff $c$ by $o(c)$. Note that $o(c)$ is equivalent to

$$
\begin{aligned}
& l(c) \int_{0}^{c} K(t) f(t) d t+h(c) \int_{c}^{1} K(t) f(t) d t \\
& =h(c) \mathbb{E}[K(X)]-n(c) \mathbb{E}[K(X) \mid X \leq c] \\
& =\frac{1-\mu_{1}-c \mu_{2}}{1-c} \mathbb{E}[K(X)]-\frac{1-\mu_{1}-\mu_{2}}{1-c} \mathbb{E}[K(X) \mid X \leq c] \\
& =\left(\frac{1-\mu_{1}-\mu_{2}}{1-c}+\mu_{2}\right) \mathbb{E}[K(X)]-\frac{1-\mu_{1}-\mu_{2}}{1-c} \mathbb{E}[K(X) \mid X \leq c] \\
& =\left(\frac{1-\mu_{1}-\mu_{2}}{1-c}\right)[\mathbb{E}[K(X)]-\mathbb{E}[K(X) \mid X \leq c]]+\mu_{2} \mathbb{E}[K(X)] \\
& =\left(1-\mu_{1}-\mu_{2}\right) \frac{\mathbb{E}[K(X)]-\mathbb{E}[K(X) \mid X \leq c]}{1-c}+\mu_{2} \mathbb{E}[K(X)]
\end{aligned}
$$

Define $T(x)$ as

$$
T(x)=\mathbb{E}[K(X) \mid X \leq x]
$$

It is immediate that $o(c)$ is decreasing (increasing) if and only if

$$
S(c)=\frac{T(1)-T(c)}{1-c}
$$

is decreasing (increasing). And $S$ is decreasing (increasing) if $T$ is concave (convex).

In the remainder of the proof, we analyze the three cases, where we assume $H^{\prime}(\cdot) / K^{\prime}(\cdot)$ to be constant, increasing, or decreasing.

(1) $H^{\prime}(\cdot) / K^{\prime}(\cdot)$ is constant

In this case, $H(v)=\alpha+\beta K(v)$ for some real number $\alpha$ and some positive $\beta$. Then the binding UB constraint would be rewritten as $\int_{0}^{1} f(v) K(v) q(v) d v=\frac{1-\mu_{1}-\alpha}{\beta}$. That is, the objective function must take value $\frac{1-\mu_{1}-\alpha}{\beta}$. Any feasible rule $q$ such that UB binds obtains this result. The set of such rules is not empty. For instance, any two-step rule with parameters $c \in\left[d^{*}, \frac{\mu_{1}}{1-\mu_{2}}\right], h(c)$ and $l(c)$ readily obtains $\int_{0}^{1} f(v) q(v) K(v) d v=\frac{1-\mu_{1}-\alpha}{h}$, by construction. This includes both $q^{M *}$ and $q^{M * *}$. Also, any convex combination of all these two-step rules obtains the same result.

(2) $H^{\prime}(\cdot) / K^{\prime}(\cdot)$ is increasing 
We first establish the following lemma.

Lemma 6 If $H^{\prime}(\cdot) / K^{\prime}(\cdot)$ is strictly increasing, then $T$ is strictly concave (hence $S$ is strictly decreasing).

Proof of Lemma 6 We have $H^{\prime}(c) / K^{\prime}(c)=\left(2-\frac{f^{\prime}(c) F(c)}{f(c)^{2}}\right) / K^{\prime}(c)$ and $T^{\prime}(c)=\frac{f(c)}{F(c)}[K(c)-T(c)]=$ $\frac{f(c) \int_{0}^{c} F(x) K^{\prime}(x) d x}{F(c)^{2}}$. Now, $T^{\prime \prime}(c) / T^{\prime}(c)=\frac{f^{\prime}(c)}{f(c)}-2 \frac{f(c)}{F(c)}+\frac{F(c) K^{\prime}(c)}{\int_{0}^{c} F(x) K^{\prime}(x) d x}$. Multiplying by $\frac{F(c)}{f(c) K^{\prime}(c)}$ we obtain that the sign of $T^{\prime \prime}(c)$ is the sign of $Z(c)=1 / T^{\prime}(c)-H^{\prime}(c) / K^{\prime}(c)$. Notice that $\lim _{c \rightarrow 0} Z(c)=0$ (applying l'Hôpital's rule, taking into account that $K^{\prime}(\cdot)$ is always positive). Thus around $c \rightarrow 0=0, T^{\prime}(c)$ does not vary while $H^{\prime}(c) / K^{\prime}(c)$ increases. Then, for some $\varepsilon>0$ and every $c \in(0, \varepsilon)$ we have $T^{\prime \prime}(c)<0$ (since $Z(c)<0)$. Since $Z$ is continuous on $(0,1)$, we cannot have $c^{\prime} \in(0,1)$ such that $Z\left(c^{\prime}\right)>0$. If such $c^{\prime}$ exists, then by continuity of $Z$ there is $c^{\prime \prime} \in\left(\varepsilon, c^{\prime}\right)$ such that $Z\left(c^{\prime \prime}\right)=0<Z^{\prime}\left(c^{\prime \prime}\right)$. But again $Z\left(c^{\prime \prime}\right)=0$ implies $T^{\prime \prime}\left(c^{\prime \prime}\right)=0$, and given $H^{\prime}(\cdot) / K^{\prime}(\cdot)$ is increasing, we get $Z^{\prime}\left(c^{\prime \prime}\right)<0$, which is a contradiction. Hence, $Z$ is negative on the domain $(0,1)$; thus $T$ is strictly concave (and $S$ is decreasing).

Then, among the two-step rules, the one depicted in the proposition, $q^{M *}$, is optimal. In the rest of the proof we show that no other rule such that both MC and UB bind can obtain higher welfare.

In order to do so, we first make a change of variables, making use of $K(\cdot)$ constituting a one-to-one mapping. Let $k=K(v), \varphi(k)=f\left(K^{-1}(k)\right) \cdot K^{-1 \prime}(k)$ and $\rho(k)=q\left(K^{-1}(k)\right)$. Then each rule $q$ can be rewritten as a rule $\rho:[K(0), K(1)] \rightarrow[0,1]$ that has an associated density function $r(v)=\frac{\rho(v) \varphi(v)}{\mu_{2}}$. Indeed, MC implies $\int_{K(0)}^{K(1)} r(k) d k=1 . R(k)=\int_{K(0)}^{k} r(t) d t$ is the distribution function associated to $\rho$. The maximization problem could be rewritten as finding a distribution function $R$, as follows:

$$
\max _{R} \mathbb{E}_{R} k \text { s.t. } \mathbb{E}_{R} \Pi(k)=\frac{1-\mu_{1}}{\mu_{2}} .
$$

where $\Pi(k)=H\left(K^{-1}(k)\right)$. Notice that $H^{\prime}(\cdot) / K^{\prime}(\cdot)$ is strictly increasing if and only if $\Pi(k)$ is convex. Moreover, $\Pi(\cdot)$ is increasing because both $H(\cdot)$ and $K^{-1}(\cdot)$ are increasing.

The problem is incomplete because $R$ should be compatible with a monotonic $q$. Yet this is irrelevant in our proof. We use the notation $R^{*}$ and $R^{* *}$ for the distributions associated with $q^{M *}$ and $q^{M * *}$, respectively. Notice that $\mathbb{E}_{R^{*}} \Pi(k)=\mathbb{E}_{R^{* *}} \Pi(k)=\frac{1-\mu_{1}}{\mu_{2}}$.

Next, we establish the following lemma.

Lemma 7 If for $R^{*} \neq R \neq R^{* *}$ we have $E_{R} k=E_{R^{*}} k$ (or $=E_{R^{* *}} k$ ), then $R$ either second-order stochastically dominates, or is dominated by, $R^{*}\left(R^{* *}\right)$.

Proof of Lemma 7 First, note that no feasible $R$ can first-order stochastically dominate, or be dominated by, either $R^{*}$ or $R^{* *}$. This is because a domination implies (since $\Pi$ is increasing) $E_{R} \Pi(k) \neq \frac{1-\mu_{1}}{\mu_{2}}$.

This implies $q(0)<l\left(\frac{\mu_{1}}{1-\mu_{2}}\right)$ for all feasible rules other than $q^{M * *}$ (otherwise $R$ would be first-order stochastically dominated by $\left.R^{* *}\right)$. Moreover, for all feasible rules other than $q^{M *}$, we have to have $q(1)>h\left(d^{*}\right)$ (otherwise $R$ would be first-order stochastically dominated by $R^{*}$ ). 
Another implication is: If $q(v)=0$ for almost all $v \leq d^{*}$ and $q \neq q^{M *}, q$ is not feasible; if $q(v)=1$ for almost all $v \geq \frac{\mu_{1}}{1-\mu_{2}}$ and $q \neq q^{M * *}, q$ is not feasible.

From the previous two lines, we can establish that if $q$ is feasible and is not either $q^{M *}$ or $q^{M * *}$, both functions $\left[r(k)-r^{*}(k)\right]$ and $\left[r(k)-r^{* *}(k)\right]$ switch signs exactly twice.

Then, if $E_{R} k=E_{R^{*}} k$, we have that $R$ either second-order dominates, or is dominated by, $R^{*}$. The same happens if $E_{R} k=E_{R^{* *}} k$.

Corollary 2 An immediate implication of Lemma 7 and the assumption that $H^{\prime}(\cdot) / K^{\prime}(\cdot)$ is strictly monotonic is that $E_{R} \Pi(k) \neq \frac{1-\mu_{1}}{\mu_{2}}$.

Finally, we have the tools to show that $q^{M *}$ is optimal. By the method of contradiction suppose that there is a feasible $q$ and its associated distribution $R$ is such that $\mathbb{E}_{R} k>\mathbb{E}_{R^{*}} k$. Since $q^{M * *}$ is strictly worse than $q^{M *}$, we have $\mathbb{E}_{R^{* *}} k<\mathbb{E}_{R^{*}} k$. Then, for some $\lambda \in(0,1)$, we can find $q^{\lambda}=\lambda q+(1-\lambda) q^{M * * 27}$ with associated distribution $R^{\lambda}=\lambda R+(1-\lambda) R^{* *}$ such that $\mathbb{E}_{R^{\lambda}} k=\mathbb{E}_{R^{*}} k$. From the previous corollary we know that $\mathbb{E}_{R^{\lambda}} \Pi(k) \neq \mathbb{E}_{R^{*}} \Pi(k)=\frac{1-\mu_{1}}{\mu_{2}}$. But since $\mathbb{E}_{R^{* *}} \Pi(k)=\frac{1-\mu_{1}}{\mu_{2}}$ and $\mathbb{E}_{R^{\lambda}} \Pi(k)=\lambda \mathbb{E}_{R} \Pi(k)+(1-\lambda) \mathbb{E}_{R^{* *}} \Pi(k)$, we obtain $\mathbb{E}_{R} \Pi(k) \neq \frac{1-\mu_{1}}{\mu_{2}}$, which is a contradiction.

(3) $H^{\prime}(\cdot) / K^{\prime}(\cdot)$ is decreasing

The proof is analogous to the case where $H^{\prime}(\cdot) / K^{\prime}(\cdot)$ is increasing, with analogous claims. In this case, we argue that if $H^{\prime}(\cdot) / K^{\prime}(\cdot)$ is decreasing, then $T$ is strictly convex (hence $S$ is strictly increasing). Thus, $q^{M * *}$ is optimal among two-step rules. To show that no other feasible rule $q$ obtains higher welfare, the analogous results apply. By method of contradiction, consider a feasible $q$ and its associated distribution $R$ be such that $\mathbb{E}_{R} k>\mathbb{E}_{R^{* *}} k$. Since $q^{M * *}$ is strictly better than $q^{M *}$, we have $\mathbb{E}_{R^{* *}} k>\mathbb{E}_{R^{*}} k$. For some $\lambda \in(0,1)$, we can define $q^{\lambda}=\lambda q+(1-\lambda) q^{*}$ with associated distribution $R^{\lambda}=\lambda R+(1-\lambda) R^{*}$ such that $\mathbb{E}_{R^{\lambda}} k=\mathbb{E}_{R^{* *}} k$. We have that $R^{\lambda}$ either second-order dominates, or is dominated by, $R^{* *}$. Hence we know that $\mathbb{E}_{R^{\lambda}} \Pi(k) \neq \mathbb{E}_{R^{* *}} \Pi(k)=\frac{1-\mu_{1}}{\mu_{2}}$. But since $\mathbb{E}_{R^{*}} \Pi(k)=\frac{1-\mu_{1}}{\mu_{2}}$ and $\mathbb{E}_{R^{\lambda}} \Pi(k)=\lambda \mathbb{E}_{R} \Pi(k)+(1-\lambda) \mathbb{E}_{R^{*}} \Pi(k)$, we obtain $\mathbb{E}_{R} \Pi(k) \neq \frac{1-\mu_{1}}{\mu_{2}}$, which is a contradiction.

Proof of Corollary 1. For the proof of this Corollary, we focus on point (1) of the Proposition. Take $\Omega(v)=\frac{1}{1+f(1)}[f(v) v+F(v)]$. We have $\Omega(\cdot) \geq 0, \Omega(1)=1$ and, under Assumption $3, \Omega^{\prime}(\cdot) \geq 0(\Omega$ is a proper weight structure). Moreover, the corresponding $\Omega$-virtual valuation is $K(v)=\frac{f(1)}{1+f(1)} H(v)$ which under Assumption 1 is increasing (that is, it meets Assumption 2). Since $K(v)$ is linear in $H(v), H^{\prime}(\cdot) / K^{\prime}(\cdot)$ is constant, thus all IC feasible rules where UB binds solve the problem given $\Omega$.

Proof of Proposition 3. First, in the simple maximization problem (13) above, the "benefit to cost ratio" of high positions is $\frac{1}{\pi_{1}}$, whereas it is $\frac{v}{\pi_{2}}$ for the medium positions. Therefore, as long as the constraint $p+q \leq 1$ is not binding, agents with $v<v^{*}\left(\pi_{1}, \pi_{2}\right) \equiv \frac{\pi_{2}}{\pi_{1}}$ would want to buy from high positions, and agents with $v>v^{*}\left(\pi_{1}, \pi_{2}\right) \equiv \frac{\pi_{2}}{\pi_{1}}$ would want to buy from medium positions. We already argued that $\pi_{1} \geq 1$, if we also have $\pi_{2} \geq 1$, then agents would have no money left for buying inferior positions (and $p+q \leq 1$

${ }^{27}$ Note that any convex combination of two feasible mechanisms is also feasible. 
does not bind). We present this case as Case 1 below, which turns out to correspond to the tough market. On the other hand, if we have $\pi_{2}<1$, then agents with $v>\frac{\pi_{2}}{\pi_{1}}$ would have money left after buying the whole probability of medium positions $(q(v)=1)$. We present this case as Case 2 below, which turns out to correspond to the mild market.

\section{Case 1. medium-class positions are expensive: $\pi_{2}^{*} \geq 1$.}

Suppose $\pi_{2}^{*} \geq 1$ in a CE, and let the unique indifferent type be $v^{*}$ (which will be $v^{*}\left(\pi_{1}, \pi_{2}\right)$ equal to $\left.\frac{\pi_{2}}{\pi_{1}}\right)$. We can observe that types below $v^{*}$ buy only high positions $\left(p=\frac{1}{\pi_{1}}, q=0\right)$, and types above $v^{*}$ buy only medium positions $\left(p=0, q=\frac{1}{\pi_{2}}\right)$. For this to be a CE, we have to have

$$
\frac{F\left(v^{*}\right)}{\pi_{1}}=\mu_{1}
$$

and

$$
\frac{1-F\left(v^{*}\right)}{\pi_{2}}=\mu_{2} .
$$

These two imply

$$
\frac{\pi_{2}}{\pi_{1}}=\frac{\frac{1-F\left(v^{*}\right)}{\mu_{2}}}{\frac{F\left(v^{*}\right)}{\mu_{1}}} .
$$

Since $\frac{\pi_{2}}{\pi_{1}}=v^{*}\left(\pi_{1}, \pi_{2}\right)$, we can conclude that a CE can exist only if there is a type $v^{*} \in(0,1)$ such that

$$
\frac{F\left(v^{*}\right) v^{*}}{1-F\left(v^{*}\right)}=\frac{\mu_{1}}{\mu_{2}} .
$$

It is immediate that this type exists and is unique. This is because $\frac{F(v) v}{1-F(v)}$ is strictly increasing with range $[0, \infty)$. However, this does not mean that this type of a CE necessarily exists. One must check the condition $\pi_{2}^{*} \geq 1$, or $\mu_{2} \leq 1-F\left(v^{*}\right)$, or $v^{*} \leq F^{-1}\left(1-\mu_{2}\right)$. Given that the LHS of equation (30) is monotonically increasing, this CE exists if and only if

$$
\frac{\left(1-\mu_{2}\right) F^{-1}\left(1-\mu_{2}\right)}{\mu_{2}} \geq \frac{\mu_{1}}{\mu_{2}}
$$

or $\mu_{1}-\left(1-\mu_{2}\right) F^{-1}\left(1-\mu_{2}\right) \leq 0$, which is exactly when the economy is "tough."

Hence, in a tough market, the CE prices are

$$
\begin{aligned}
& \pi_{1}^{*}=\frac{F\left(v^{*}\right)}{\mu_{1}} \\
& \pi_{2}^{*}=\frac{1-F\left(v^{*}\right)}{\mu_{2}}
\end{aligned}
$$

where $v^{*}$ uniquely solves (30).

In this CE, the assignment of probabilities is as follows. Types $v<v^{*}$ obtain the probability bundle

$$
\left(\frac{\mu_{1}}{F\left(v^{*}\right)}, 0,1-\frac{\mu_{1}}{F\left(v^{*}\right)}\right),
$$


and types $v>v^{*}$ get

$$
\left(0, \frac{\mu_{2}}{1-F\left(v^{*}\right)}, 1-\frac{\mu_{2}}{1-F\left(v^{*}\right)}\right) .
$$

where the first, second, and third components represents probability shares of high, medium and low positions, respectively.

\section{Case 2. medium-class positions are cheap: $\pi_{2}^{*}<1$.}

Suppose $\pi_{2}^{*}<1$ in a CE, and let the unique indifferent type be $v^{*}$. Types below $v^{*}$ buy only high positions $\left(p=\frac{1}{\pi_{1}}, q=0\right)$. On the other hand, types above $v^{*}$ buy medium positions, and buy high positions with the rest of their money in such a way that the total probability of being assigned high or medium positions does not exceed $1(p+q \leq 1)$. The solution to the high types' maximization problem are given by $p=\frac{1-\pi_{2}}{\pi_{1}-\pi_{2}}$ and $q=\frac{\pi_{1}-1}{\pi_{1}-\pi_{2}} \cdot{ }^{28}$ Also, as in Case 1 , we can argue that the unique indifferent type $v^{*}\left(\pi_{1}, \pi_{2}\right)$ is again equal to $\frac{\pi_{2}}{\pi_{1}} \cdot{ }^{29}$ For this to be a CE, we have to have

$$
\begin{aligned}
& \frac{F\left(v^{*}\right)}{\pi_{1}}+\left(1-F\left(v^{*}\right)\right) \frac{1-\pi_{2}}{\pi_{1}-\pi_{2}}=\mu_{1} \\
&\left(1-F\left(v^{*}\right)\right) \frac{\pi_{1}-1}{\pi_{1}-\pi_{2}}=\mu_{2} .
\end{aligned}
$$

The above system of two linear equations has the following unique solution in prices, as a function of $v^{*}$, $\mu_{1}$ and $\mu_{2}$.

$$
\begin{aligned}
& \pi_{1}^{*}=\frac{F\left(v^{*}\right)}{F\left(v^{*}\right)-\left(1-\mu_{1}-\mu_{2}\right)} \\
& \pi_{2}^{*}=\frac{1}{\mu_{2}} \frac{\left(1-\mu_{1}\right) F\left(v^{*}\right)-\left(1-\mu_{1}-\mu_{2}\right)}{F\left(v^{*}\right)-\left(1-\mu_{1}-\mu_{2}\right)} .
\end{aligned}
$$

Since $v^{*}=\frac{\pi_{2}}{\pi_{1}}$, we have to have

$$
v^{*}=\frac{\pi_{2}^{*}}{\pi_{1}^{*}}=\frac{1-\mu_{1}}{\mu_{2}}-\frac{1-\mu_{1}-\mu_{2}}{\mu_{2} F\left(v^{*}\right)}
$$

or

$$
\left(1-\mu_{1}-v^{*} \mu_{2}\right) F\left(v^{*}\right)=1-\mu_{1}-\mu_{2} .
$$

The question is whether there exists a $v^{*}$ that solves $(31)$, and whether for that $v^{*}$ we would have $\pi_{2}^{*}<1$.

Consider the function $G(v) \equiv\left(1-\mu_{1}-v \mu_{2}\right) F(v)-\left(1-\mu_{1}-\mu_{2}\right)$. Observe that (i) $G(0)<0$ and (ii) $G\left(F^{-1}\left(1-\mu_{2}\right)\right)=\left(1-\mu_{1}-\mu_{2} F^{-1}\left(1-\mu_{2}\right)\right)\left(1-\mu_{2}\right)-\left(1-\mu_{1}-\mu_{2}\right)>0$. The latter is due to $F^{-1}\left(1-\mu_{2}\right)<\frac{\mu_{1}}{1-\mu_{2}}$ and the fact that $\left(1-\mu_{1}-\frac{\mu_{1} \mu_{2}}{1-\mu_{2}}\right)\left(1-\mu_{2}\right)=1-\mu_{1}-\mu_{2}$. Since $G$ is continuous, the intermediate value theorem applies: there exists a $v$ with $G(v)=0$.

\footnotetext{
${ }^{28}$ The maximization problem is to maximize $p+q v$ subject to $\pi_{1} p+\pi_{2} q \leq 1$ and $p+q \leq 1$. We can argue that at the optimal solution both constraints have to bind, and $p=\frac{1-\pi_{2}}{\pi_{1}-\pi_{2}}$ and $q=\frac{\pi_{1}-1}{\pi_{1}-\pi_{2}}$ solve them simultaneously and therefore are the maximizers.

${ }^{29}$ If we compare the utility at the optimal bundle of lower types and higher types, we observe that again the critical type is $v^{*}\left(\pi_{1}, \pi_{2}\right)=\frac{\pi_{2}}{\pi_{1}}$ as $\frac{1-\pi_{2}}{\pi_{1}-\pi_{2}}+v \frac{\pi_{1}-1}{\pi_{1}-\pi_{2}}>\frac{1}{\pi_{1}}$ if and only if $v>\frac{\pi_{2}}{\pi_{1}}$.
} 
We can also show the uniqueness of $v^{*}$. We have $G^{\prime}(v)=f(v)\left(1-\mu_{1}-v \mu_{2}\right)-\mu_{2} F(v)$. Hence, its sign is equivalent to that of $G^{\prime}(v) / f(v)=1-\mu_{1}-\mu_{2} H(v)$, which is a decreasing function (since $H(\cdot)$ is increasing). We therefore conclude that $G$ is concave. Since $G$ is concave, $G(0)<0, G\left(F^{-1}\left(1-\mu_{2}\right)\right)>0$, and $G(1)=0$, we can conclude that there exists a unique $v^{*}$ that satisfies $G\left(v^{*}\right)=0$ (and also $v^{*} \in\left(0, F^{-1}\left(1-\mu_{2}\right)\right)$ ).

Finally, we show that under a mild market, we would also have $\pi_{2}^{*}<1$. This follows because

$$
\frac{1}{\mu_{2}} \frac{\left(1-\mu_{1}\right) F\left(v^{*}\right)-\left(1-\mu_{1}-\mu_{2}\right)}{F\left(v^{*}\right)-\left(1-\mu_{1}-\mu_{2}\right)}<1
$$

reduces to $1-F\left(v^{*}\right)>\mu_{2}$, or $v^{*}<F^{-1}\left(1-\mu_{2}\right)$, which we have shown. On the other hand, under a tough market we would have $G\left(F^{-1}\left(1-\mu_{2}\right)\right)>0$, which implies $v^{*} \geq F^{-1}\left(1-\mu_{2}\right)$, and $\pi_{2}^{*} \geq 1$.

In conclusion, under a mild market, we would have $\pi_{2}^{*}<1$, and in $\mathrm{CE}$, the assignment of probabilities are as follows. Types $v<v^{*}$ obtain the probability bundle

$$
\left(1-\frac{1-\mu_{1}-\mu_{2}}{F\left(v^{*}\right)}, 0, \frac{1-\mu_{1}-\mu_{2}}{F\left(v^{*}\right)}\right),
$$

and types $v>v^{*}$ get

$$
\left(1-\frac{\mu_{2}}{1-F\left(v^{*}\right)}, \frac{\mu_{2}}{1-F\left(v^{*}\right)}, 0\right)
$$

where the first, second, and third components represents the probability shares of high, medium and low positions, respectively.

Proof of Proposition 4. Obviously we must consider only cases when $m_{1} \geq \mu_{1}$ (if $m_{1}<\mu_{1}$ using strategy 2 would never be a best response), in which we say that high-class positions are overdemanded. So we are left with two cases to analyze: one in which medium-class positions are overdemanded $\left(m_{2} \geq \mu_{2}\right)$, and the case in which they are underdemanded $\left(m_{2}<\mu_{2}\right)$. It turns out that the former case corresponds to the tough market and the latter to the mild market.

Case 1. medium-class positions are overdemanded: $m_{2} \geq \mu_{2}$.

In this case it is clear that those who are rejected in the first round end up being assigned to low-class positions. Those choosing strategy $j \in\{1,2\}$ obtain $\mu_{j} / m_{j}$ chances at the position ranked first and $1-\mu_{j} / m_{j}$ chances for a low-class position. An indifferent type $v^{*}\left(m_{1}, m_{2}\right)$ meets $\mu_{1} / m_{1}=v^{*}\left(m_{1}, m_{2}\right) \mu_{2} / m_{2}$. Types above the indifferent type best-respond by choosing strategy 2 , and types below choose strategy 1 . Therefore, a Nash equilibrium (NE) is characterized by the existence of a $v^{*} \in(0,1)$ such that

$$
\frac{F\left(v^{*}\right) v^{*}}{1-F\left(v^{*}\right)}=\frac{\mu_{1}}{\mu_{2}}
$$

As in Case 1 of the proof of Proposition 3, a solution to this equation exists and is unique. It remains be shown that in effect $m_{2}>\mu_{2}$, or $\mu_{2}<1-F\left(v^{*}\right)$, or $v^{*}<F^{-1}\left(1-\mu_{2}\right)$. Given that the LHS of the equation above is monotonically increasing, a Case $1 \mathrm{NE}$ exists if and only if

$$
\frac{\left(1-\mu_{2}\right) F^{-1}\left(1-\mu_{2}\right)}{\mu_{2}}>\frac{\mu_{1}}{\mu_{2}}
$$


or $\mu_{1}-\left(1-\mu_{2}\right) F^{-1}\left(1-\mu_{2}\right) \leq 0$, which exactly is when the market is "tough."

In this Bayesian Nash equilibrium, Types $v<v^{*}$ obtain the probability bundle

$$
\left(\frac{\mu_{1}}{F\left(v^{*}\right)}, 0,1-\frac{\mu_{1}}{F\left(v^{*}\right)}\right)
$$

whereas types $v>v^{*}$ get

$$
\left(0, \frac{\mu_{2}}{1-F\left(v^{*}\right)}, 1-\frac{\mu_{2}}{1-F\left(v^{*}\right)}\right) .
$$

Note that in Cases 1, both the Pseudomarket (CE) and the Boston mechanism (NE) obtain the same random assignment. This is not a coincidence. Miralles (2008) shows that both mechanisms obtain the same assignment in cases where only one kind of position is underdemanded.

Case 2. medium-class positions are underdemanded: $m_{2}<\mu_{2}$.

In this case, those agents who play strategy 2 are assigned to medium-class positions with certainty. Agents playing strategy 1 obtain $\frac{\mu_{1}}{m_{1}}$ chances at the high-class positions in the first assignment round, $\frac{\mu_{2}-m_{2}}{m_{1}}$ chances (in the second round) for medium-class positions, and $\frac{1-\mu_{1}-\mu_{2}}{m_{1}}$ for the low-class positions in the last round.

Let $v^{*}$ be the type who is indifferent between the two strategies. We then have

$$
v^{*}=\frac{\mu_{1}}{m_{1}}+\frac{\mu_{2}}{m_{1}} v^{*}
$$

and hence

$$
v^{*}=\frac{\mu_{1}}{m_{1}-\mu_{2}+m_{2}}=\frac{\mu_{1}}{1-\mu_{2}}
$$

(since $\left.m_{1}+m_{2}=1\right)$.

Again, in equilibrium, types $v>v^{*}\left(m_{1}, m_{2}\right)=\frac{\mu_{1}}{1-\mu_{2}}$ choose strategy 2 , and types $v<\frac{\mu_{1}}{1-\mu_{2}}$ choose strategy 1 . Hence, $m_{1}=F\left(\frac{\mu_{1}}{1-\mu_{2}}\right)$ and $m_{2}=1-F\left(\frac{\mu_{1}}{1-\mu_{2}}\right)$.

We now check that $m_{2}<\mu_{2}$ holds. That is, $1-F\left(\frac{\mu_{1}}{1-\mu_{2}}\right)<\mu_{2}$. This is equivalent to the market being "mild" $\mu_{1}-\left(1-\mu_{2}\right) F^{-1}\left(1-\mu_{2}\right)>0$.

Finally, the obtained probability bundles are

$$
\left(\frac{\mu_{1}}{F\left(\frac{\mu_{1}}{1-\mu_{2}}\right)}, 1-\frac{1-\mu_{2}}{F\left(\frac{\mu_{1}}{1-\mu_{2}}\right)}, \frac{1-\mu_{1}-\mu_{2}}{F\left(\frac{\mu_{1}}{1-\mu_{2}}\right)}\right)
$$

for types $v>\frac{\mu_{1}}{1-\mu_{2}}$, and

for types $v<\frac{\mu_{1}}{1-\mu_{2}}$.

\section{Appendix B: Varying Ordinal Preferences}


Define $U(v)=a p(v)+q(v) v$. We first establish the following payoff equivalence result with the standard arguments.

Lemma 8 Incentive compatibility is equivalent to

$$
U(v)=a p(0)+\int_{0}^{v} q(t) d t
$$

and $q$ increasing, $p$ decreasing, $q+p$ increasing for $v \in[0, a]$ and decreasing for $v \in[a, 1]$ (together called monotonicity conditions).

Note that the extended problem becomes more complex than the original one since UB applies to $a$ instead of the highest type: we need to ensure that $p(a)+q(a) \leq 1$.

Utilitarian social welfare can be shown to be

$$
U S W=a \mu_{1}+\int_{0}^{1} q(v) v f(v) d v
$$

Rawlsian social welfare is equal to

$$
R S W=a p(0)
$$

We establish the following result. The proof is similar to the proof of Proposition 2.

Proposition 5 A rule $(p(\cdot), q(\cdot))$ is incentive compatible and feasible if and only if

$$
p(v)=\mu_{1}+\frac{1}{a}\left(\int_{0}^{1} J(t) q(t) f(t) d t+\int_{0}^{v} q(t) d t-q(v) v\right),
$$

and

$$
\begin{aligned}
\int_{0}^{1} q(t) f(t) d v & =\mu_{2} \\
\int_{0}^{a} H(t) q(t) f(t) d t+\int_{a}^{1} J(t) q(t) f(t) d t & \leq a-a \mu_{1} \\
\int_{0}^{1} H(t) q(t) f(t) d t & \geq q(1)-a \mu_{1} \\
q(0) & \geq 0 \text { and } q \text { is increasing. }
\end{aligned}
$$

The first constraint is market-clearing (MC), the second one is the upper bound (UB), and the third one is the lower bound (LB).

We can easily see that, as in the original model, the social welfare maximand is equivalent to

$$
\int_{0}^{1} q(t) K(t) f(t) d t
$$

subject to the above MC, UB, LB. 
In the relaxed problem, in which we consider only MC (and ignore UB and LB), since the objective functions and $\mathrm{MC}$ are the same, Lemma 3 holds and the solution to the relaxed problem is given by

$$
q^{*}(t)=\left\{\begin{array}{cc}
1 & t \in\left[v^{*}, 1\right] \\
0 & t \in\left[0, v^{*}\right)
\end{array}\right.
$$

for $v^{*}=F^{-1}\left(1-\mu_{2}\right)$.

Since we assume $a \geq H\left(F^{-1}\left(1-\mu_{2}\right)\right)\left(>F^{-1}\left(1-\mu_{2}\right)\right)$, the optimal rule in the relaxed problem satisfies UB if and only if

$$
\begin{aligned}
a-a \mu_{1} & \geq \int_{F^{-1}\left(1-\mu_{2}\right)}^{a} H(t) f(t) d t+\int_{a}^{1} J(t) f(t) d t \\
& =a-\left(1-\mu_{2}\right) F^{-1}\left(1-\mu_{2}\right)
\end{aligned}
$$

or

$$
a \mu_{1}-\left(1-\mu_{2}\right) F^{-1}\left(1-\mu_{2}\right) \leq 0
$$

As in our main model, we call an economy a tough market if this condition is satisfied with strict inequality. If it is violated, we call the economy to be a mild market. Note that in this extension, a tough market is more likely: the condition in the main model required $\left(1-\mu_{2}\right) F^{-1}\left(1-\mu_{2}\right)$ to be greater than $\mu_{1}$; in the extension it should only be greater than $a \mu_{1}$. This brings us to the first fact we stated above.

As for the second statement, we first observe that when $a \geq H\left(F^{-1}\left(1-\mu_{2}\right)\right)$, in a maximization problem where the objective function is $\int_{0}^{a} H(t) q(t) f(t) d t+\int_{a}^{1} J(t) q(t) f(t) d t$ and the constraint is just MC, the solution is $q^{*}$. This is because $E[J(t) \mid t \geq v]=v$ and, therefore, in the optimal solution, $q$ has to be constant between $b \equiv H^{-1}(a)$ and 1 . Given this, and also $b>F^{-1}\left(1-\mu_{2}\right)$, one can easily argue that the highest value that the LHS of the UB constraint can get is achieved by $q^{*}$, and this means that in a tough market UB never binds. With this simplifying insight, the proof of the following proposition follows the same steps as the proof of Proposition 1, and it is omitted.

Proposition 6 For $a \geq H\left(F^{-1}\left(1-\mu_{2}\right)\right)$, in a tough market, the optimal rule is

$$
q^{a}(t)= \begin{cases}\frac{\mu_{1}}{F\left(v^{a}\right) v^{a}} & t \in\left[v^{a}, 1\right] \\ 0 & t \in\left[0, v^{a}\right)\end{cases}
$$

for $v^{a}$ that uniquely solves

$$
\frac{F(v) v}{1-F(v)}=\frac{a \mu_{1}}{\mu_{2}}
$$

The next main result follows immediately. It stems from similar arguments as in Proposition 3, Proposition 4, and Theorem 1.

Proposition 7 For $a \geq H\left(F^{-1}\left(1-\mu_{2}\right)\right)$, in a tough market, the optimal assignments are implemented by both $P M$ and $B M$. 
As for mild markets, we can get results in this extension similar to the ones in our main model. For mild markets, it turns out that under the same conditions as in Theorem 1, BM continues to be optimal. Yet sufficient conditions for PM being optimal in Theorem 1 need to be strengthened. ${ }^{30}$ Hence, in a way, this extension brings a bias in favor of BM rather than PM.

\section{Appendix C: The role of concavity/convexity of virtual valua- tions}

In this Appendix, we provide an intuition on the role that the concavity/convexity of $H$ with respect to $K$ (that is, whether $H^{\prime} / K^{\prime}$ is monotonic) plays. To simplify the explanation, we first consider the utilitarian problem, where $K(v)=v$. Then $H^{\prime} / K^{\prime}$ is monotonic if and only if $H$ is either concave or convex. Observe that the curvature of $H$ has nothing to do with the "strength" of $F$. A clear example is the case in which $H$ is linear: all power distribution functions $v^{\alpha}$, no matter how big or small $\alpha$ is, give linear $H$. Then one may wonder why is the curvature of $H$ important for our results in mild markets.

For the purpose of clarity, let us simplify the model by simply considering three points in the support of types: $v_{1}<v_{2}<v_{3}$. Let us also make $f$ constant and normalize it to $\mu_{2} / 3$. The discrete version of the maximization problem we solve is:

$$
\max \sum_{i=1}^{3} q_{i} v_{i}
$$

subject to

$$
\begin{gathered}
\sum_{i=1}^{3} q_{i}=1 \\
\sum_{i=1}^{3} H_{i} q_{i}=k
\end{gathered}
$$

and monotonicity: $q_{1} \leq q_{2} \leq q_{3}$.

Notice that, apart from monotonicity, this would be a consumer's problem in a pseudomarket à la Hylland and Zeckhauser (1979) where bundles are probability distributions. The second constraint is the budget constraint: $H_{i}$ is the price for "buying" more probability units of (assigning more $q_{i}$ to) type $i$. Indeed, in our base model $H(v)$ is well-known as the informational cost of type $v$. Let us observe possible constraints of the problem in the following figure.

\footnotetext{
${ }^{30}$ For two-step assignment rules with cutoff $c$ and UB binding, in this extension the objective function becomes $S(c)$ multiplied by a factor $(1-c) /(a-c)$. This factor is increasing in $c$, and this brings the bias in favor of higher $c$.
} 


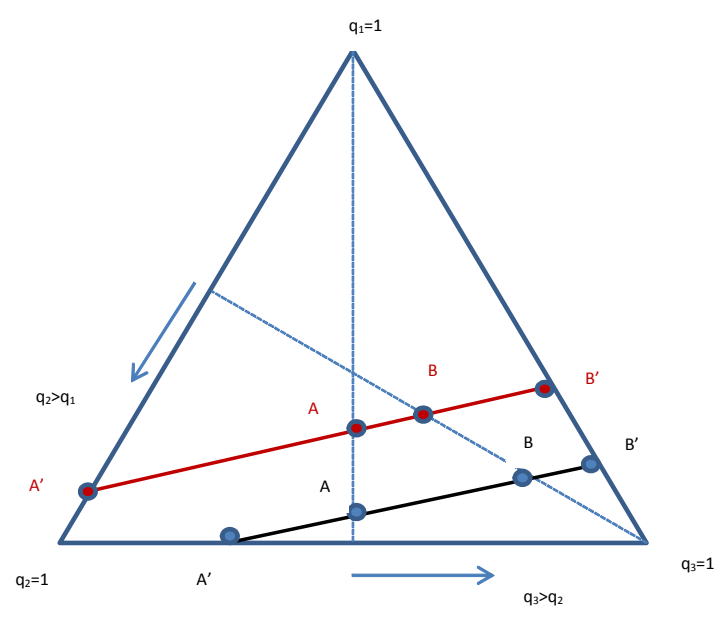

Figure 1

In the linear maximization problem of Figure 1, generically the optimum is unique and it is either A or B. Notice that, if monotonicity of $q$ were not required, the decision maker would choose either A' or B'. It turns out that one optimally chooses A (B) in the original problem if and only if she chooses A' (B') in the problem without monotonicity. Let us momentarily ignore the monotonicity constraint.

By monotonicity of $H$, we know that $H_{1}<H_{2}<H_{3}$. The budget constraint implies that $H_{1}<k<H_{3}$. We consider two different cases. In case $1, H_{2}<k$ (black budget line in figure 1). Then one has to choose between buying a distribution with support for types 3 and 2 (A'), or buying a bundle supported for types 3 and 1 (B'). If one chooses $\mathrm{A}^{\prime}$ she obtains $q_{2}^{A^{\prime}}=\frac{H_{3}-k}{H_{3}-H_{2}}$, while if she chooses B' she obtains $q_{1}^{B^{\prime}}=\frac{H_{3}-k}{H_{3}-H_{1}}$. $\mathrm{A}^{\prime}$ is preferred to $\mathrm{B}^{\prime}$ if

$$
q_{2}^{A^{\prime}} v_{2}+\left(1-q_{2}^{A^{\prime}}\right) v_{3}>q_{1}^{B^{\prime}} v_{1}+\left(1-q_{1}^{B^{\prime}}\right) v_{3}
$$

or

$$
q_{2}^{A^{\prime}}\left(v_{3}-v_{2}\right)<q_{1}^{B^{\prime}}\left(v_{3}-v_{1}\right)
$$

or

$$
\frac{H_{3}-H_{2}}{v_{3}-v_{2}}>\frac{H_{3}-H_{1}}{v_{3}-v_{1}} .
$$

From the last inequality, we can see the importance of concavity/convexity of $H$ with respect to $v$ in this decision. A' is preferred to $\mathrm{B}^{\prime}$ if the slope of $H$ with respect to $v$ decreases when the bottom valuation decreases from $v_{2}$ to $v_{1}$. That is, when information costs decrease "slower" than the bottom valuation does. In other words, when $H$ is convex. Naturally, concavity would have driven the opposite choice.

In case 2 we have $H_{2} \geq k$ (in red in the figure). In such a case, one has to choose between buying a distribution with support for types 2 and 1 (A'), or buying a bundle supported for types 3 and 1 (B'). If one chooses A' she obtains $q_{2}^{A^{\prime}}=\frac{k-H_{1}}{H_{2}-H_{1}}$, while if she chooses B' she obtains $q_{3}^{B^{\prime}}=\frac{k-H_{1}}{H_{3}-H_{1}}$. A' is preferred to B' if

$$
q_{2}^{A^{\prime}} v_{2}+\left(1-q_{2}^{A^{\prime}}\right) v_{1}>q_{3}^{B^{\prime}} v_{3}+\left(1-q_{3}^{B^{\prime}}\right) v_{1}
$$


or

$$
q_{2}^{A^{\prime}}\left(v_{2}-v_{1}\right)>q_{3}^{B^{\prime}}\left(v_{3}-v_{1}\right)
$$

or

$$
\frac{H_{2}-H_{1}}{v_{2}-v_{1}}<\frac{H_{3}-H_{1}}{v_{3}-v_{1}}
$$

Again the concavity/convexity of $H$ is crucial. A' is preferred to $\mathrm{B}$ ' if the slope of $H$ with respect to $v$ increases when the top valuation increases from $v_{2}$ to $v_{3}$. That is, when information costs increase "faster" than the top valuation does. Or in other words, when $H$ is convex. Once again, concavity would have driven the opposite choice. This is not an artifact of having only three types: we can use the same argument with a continuum of types.

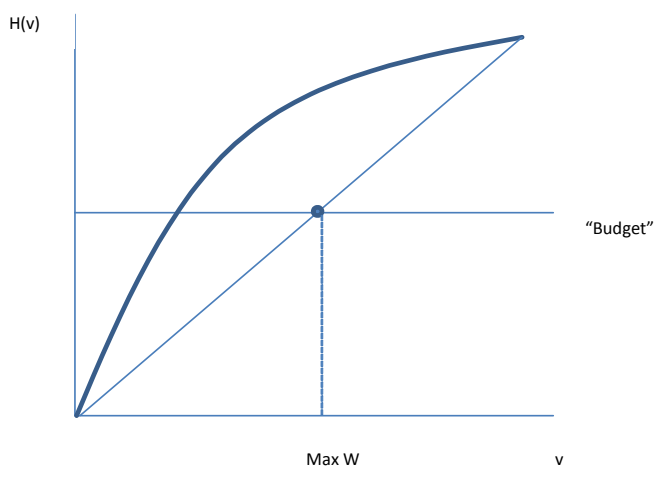

Figure 2

In Figure 2 we have a concave virtual valuation structure. Taking the extreme values 0 and 1 and drawing a segment uniting $H(0)$ and $H(1)$ gives us a convex combination of both $(q(0)+q(1)=1)$ that maximizes the valuation of the purchased bundle, subject to the "budget" constraint. An opposite argument would work under convexity: one would tend to concentrate purchases on $\tilde{v}$ such that $H(\tilde{v})$ equals the budget limit.

Going back to the original problem with monotonicity constraints, one optimally chooses a bundle that puts more weight on middle types and less on extreme types ( $\mathrm{A}$ in Figure 1 ) if $H$ is convex. The opposite happens if $H$ is concave: one tends to put more weight on extreme types and less on middle types (B in Figure 1) if $H$ is concave. In fact, the solution under convexity generates the density function $\frac{f(v) q(v)}{\mu_{2}}$ with thinnest tails among all valid assignment rules. The solution under concavity generates the density function with thickest tails instead.

The general problem is more elaborate, but the intuition is similar. Sufficient conditions are related to the curvature of $H$ with respect to $K$, that is, whether $\frac{d H(v)}{d K(v)}$ is increasing or decreasing in $v$. 


\section{Appendix D: Not every optimal rule with $K$ increasing is a two-step rule}

We provide a counterexample. We consider $F(t)=t$, and the following cumulative weight function: $\Omega(t)=$ $2 t-2|t-1 / 2|(t-1 / 2)-1 / 2$. This leads to an $\Omega$-virtual valuation function $K(t)=2|t-1 / 2|(t-1 / 2)+1 / 2$.

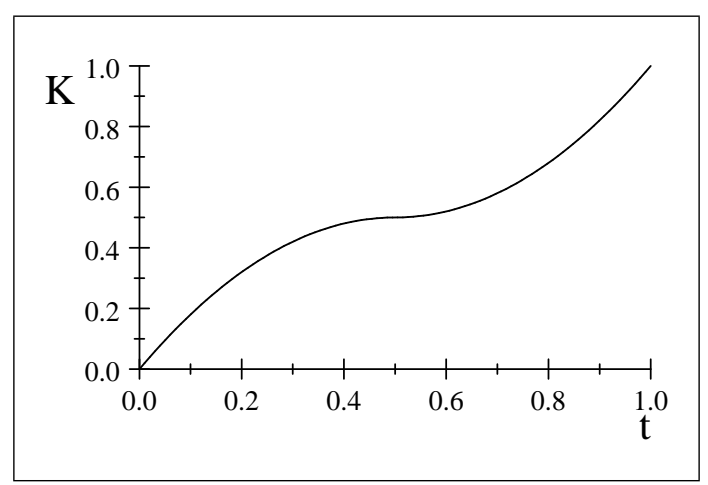

Figure 3

We set supply parameters $\mu_{1}=0.3$ and $\mu_{2}=0.6$ so that the economy is mild. In this setup, the PM equilibrium allocation rule is a two-step rule with cutoff $1 / 6$. The BM equilibrium allocation rule is a twostep rule with cutoff $3 / 4$. Notice that $H^{\prime}(t) / K^{\prime}(t)$ is increasing for $t<1 / 2$ and decreasing for $t>1 / 2$. Then sufficient conditions for the optimality of either BM or PM (Proposition 2) do not apply here.

All feasible two-step rules with both $\mathrm{MC}$ and UB binding have cutoff $c \in[1 / 6,3 / 4]$. We use the terminology that we can find in the proof of Proposition 2. Denoting

$$
T(c)=\frac{\int_{0}^{c} K(t) d t}{c}
$$

for each two-step rule with cutoff $c$, welfare is $W(c)=\left(1-\mu_{1}-\mu_{2}\right) S(c)+\mu_{2} T(1)$ (see again the proof of Proposition 2) with

$$
S(c)=\frac{T(1)-T(c)}{1-c}
$$

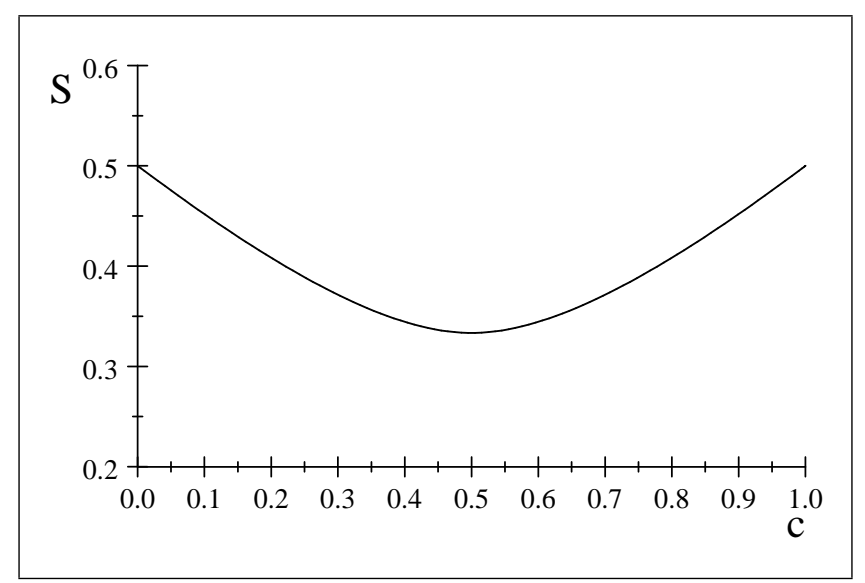

Figure 4

Notice that since $S(c)$ is U-shaped, extreme values for $c$ would be optimal. However, that would lead to non-valid allocation probabilities. For cutoffs above $3 / 4, q(1)$ would exceed 1 . For cutoffs below $1 / 6$, 
$q(0)$ would be negative. Among all valid two-step rules, the optimal one has minimum cutoff $(1 / 6$, the PM allocation rule) with associated welfare $0.1 S(1 / 6)+0.3=0.34222$. This rule is

$$
q^{P M}(t)=\left\{\begin{array}{c}
0 \text { if } t<1 / 6 \\
0.72 \text { if } t \geq 1 / 6
\end{array}\right.
$$

However, if we consider for instance a non-valid two-step rule with cutoff 0.9 , welfare would be higher: $0.1 S(0.9)+0.3=0.34519$. The problem is that the rule would be

$$
q^{0.9}(t)=\left\{\begin{array}{c}
22 / 45 \text { if } t<0.9 \\
1.6 \text { if } t \geq 0.9
\end{array}\right.
$$

with one allocation probability exceeding 1 . Nevertheless one can create the following three-step rule

$$
q^{\prime}=\frac{15}{22} q^{P M}+\frac{7}{22} q^{0.9}
$$

The rule $q^{\prime}$ is non-decreasing, positive and not exceeding 1 . Moreover, since both $q^{P M}$ and $q^{0.9}$ meet UB and MC, the same holds for $q^{\prime}$ as well. Hence $q^{\prime}$ is feasible. Clearly $q^{\prime}$ improves welfare upon the best valid two-step rule. 